\title{
DUALITY RESULTS IN BANACH AND QUASI-BANACH SPACES OF HOMOGENEOUS POLYNOMIALS AND APPLICATIONS
}

\author{
VINÍCIUS V. FÁVARO AND DANIEL PELLEGRINO
}

\begin{abstract}
Spaces of homogeneous polynomials on a Banach space are frequently equipped with quasinorms instead of norms. In this paper we develop a technique to replace the original quasi-norm by a norm in a dual preserving way, in the sense that the dual of the space with the new norm coincides with the dual of the space with the original quasi-norm. Applications to problems on the existence and approximation of solutions of convolution equations and on hypercyclic convolution operators on spaces of entire functions are provided.
\end{abstract}

\section{Contents}

1. Introduction

2. Main results

3. Prerequisites for the applications

3.1. Hypercyclicity results

3.2. Existence and approximation results 12

4. Applications

4.1. Lorentz nuclear and summing polynomials: the basics

4.2. New hypercyclic, existence and approximation results

Mathematics Subject Classifications (2010): 46A20, 46G20, 46A16, 46G25, 47A16.

Key words: Banach and quasi-Banach spaces, homogeneous polynomials, entire functions, convolution operators, Lorentz sequence spaces.

\section{INTRODUCTION}

In the 1960s, many researchers began the study of spaces of holomorphic functions defined on infinite dimensional complex Banach spaces. In this context spaces of $n$-homogeneous polynomials play a central role in the development of the theory. Several tools, such as topological tensor products and duality theory, are useful and important when we are working with spaces of homogeneous polynomials. Many duality results on spaces of homogeneous polynomials and their applications have appeared in the last decades (see for instance [5, 8, 9, 10, 11, 12, 15, 20, 22, 26, 28, 29, 33, 34, 41, 46, among others). In this paper we develop a new technique in the duality theory of spaces of homogeneous polynomials and we give some applications.

Let $E$ be a complex Banach space, $n \in \mathbb{N}$ and $\mathcal{P}\left({ }^{n} E\right)$ be the Banach space of all continuous $n$ homogeneous polynomials from $E$ to $\mathbb{C}$ with its usual norm. Suppose that $\left(\mathcal{P}_{\Delta}\left({ }^{n} E\right),\|\cdot\|_{\Delta}\right)$ is a quasinormed space of $n$-homogeneous polynomials on $E$ such that the inclusion $\mathcal{P}_{\Delta}\left({ }^{n} E\right) \hookrightarrow \mathcal{P}\left({ }^{n} E\right)$ is continuous and $\mathcal{P}_{f}\left({ }^{n} E\right) \subset \mathcal{P}_{\Delta}\left({ }^{n} E\right)$, where $\mathcal{P}_{f}\left({ }^{n} E\right)$ denotes the subspace of $\mathcal{P}\left({ }^{n} E\right)$ of all polynomials of finite

The first named author is supported by FAPESP Grant 2014/50536-7; FAPEMIG Grant PPM-00086-14; and CNPq Grants 482515/2013-9, 307517/2014-4.

The second named author is supported by CNPq. 
type. Let $C_{\Delta_{n}}>0$ be such that $\|P\| \leq C_{\Delta_{n}}\|P\|_{\Delta}$, for all $P \in \mathcal{P}_{\Delta}\left({ }^{n} E\right)$. Suppose that the normed space $\left(\mathcal{P}_{\Delta^{\prime}}\left({ }^{n} E^{\prime}\right),\|\cdot\|_{\Delta^{\prime}}\right) \subset \mathcal{P}\left({ }^{n} E^{\prime}\right)$ is such that the Borel transform

$$
\mathcal{B}:\left(\mathcal{P}_{\Delta}\left({ }^{n} E\right)^{\prime},\|\cdot\|\right) \rightarrow\left(\mathcal{P}_{\Delta^{\prime}}\left({ }^{n} E^{\prime}\right),\|\cdot\|_{\Delta^{\prime}}\right)
$$

given by $\mathcal{B}(T)(\varphi)=T\left(\varphi^{n}\right)$, for all $\varphi \in E^{\prime}$ and $T \in \mathcal{P}_{\Delta}\left({ }^{n} E\right)^{\prime}$, is a topological isomorphism. In this paper we develop a technique to construct a norm in $\mathcal{P}_{\Delta}\left({ }^{n} E\right)$ in such way that this normed space (or its completion denoted by $\left.\left(\mathcal{P}_{\widetilde{\Delta}}\left({ }^{n} E\right),\|\cdot\|_{\widetilde{\Delta}}\right)\right)$ preserves the duality given by the Borel transform, that is the topological isomorphism given by the Borel transform is still valid when we use $\mathcal{P}_{\widetilde{\Delta}}\left({ }^{n} E\right)$ instead of $\mathcal{P}_{\Delta}\left({ }^{n} E\right)$. As applications of this result, we prove that, under suitable conditions, $\left(\mathcal{P}_{\widetilde{\Delta}}\left({ }^{n} E\right)\right)_{n=0}^{\infty}$ is a holomorphy type and we provide new examples of hypercyclic convolution operators and new existence and approximation results for convolution equations.

The paper is organized as follows:

In Section 2 we develop the general theory to obtain a norm in the quasi-normed space $\mathcal{P}_{\Delta}\left({ }^{n} E\right)$ and to keep the duality via Borel transform. We also prove some technical results needed to the applications.

In Section 3 we present background results that will be needed in the next section.

In Section 4 we obtain the aforementioned applications in a case that was not possible before. We use as a prototype of model the class of Lorentz nuclear polynomials.

Throughout the paper $\mathbb{N}$ denotes the set of positive integers and $\mathbb{N}_{0}$ denotes the set $\mathbb{N} \cup\{0\}$. The letters $E$ and $F$ will always denote complex Banach spaces and $E^{\prime}$ represents the topological dual of $E$ and $E^{\prime \prime}$ its bidual. The Banach space of all continuous $m$-homogeneous polynomials from $E$ into $F$ endowed with its usual sup norm is denoted by $\mathcal{P}\left({ }^{m} E ; F\right)$. The subspace of $\mathcal{P}\left({ }^{m} E ; F\right)$ of all polynomials of finite type is represented by $\mathcal{P}_{f}\left({ }^{m} E ; F\right)$. The linear space of all entire mappings from $E$ into $F$ is denoted by $\mathcal{H}(E ; F)$. When $F=\mathbb{C}$ we write $\mathcal{P}\left({ }^{m} E\right), \mathcal{P}_{f}\left({ }^{m} E\right)$ and $\mathcal{H}(E)$ instead of $\mathcal{P}\left({ }^{m} E ; \mathbb{C}\right), \mathcal{P}_{f}\left({ }^{m} E ; \mathbb{C}\right)$ and $\mathcal{H}(E ; \mathbb{C})$, respectively. For the general theory of homogeneous polynomials and holomorphic functions we refer to Dineen [19] and Mujica [48. If $G$ and $H$ are vector spaces and $\langle\cdot, \cdot\rangle$ is a bilinear form on $G \times H$, we denote by $(G, H,\langle\cdot, \cdot\rangle)$ (or $(G, H)$ for short) the dual system. We denote by $\sigma(G, H)$ the weak topology with respect to the dual system $(G, H)$, that is, the coarsest topology on $G$ for which the linear forms $x \rightarrow\langle x, y\rangle, y \in H$ are continuous.

\section{MAin RESUlts}

Let $n \in \mathbb{N}$ and suppose that $\left(\mathcal{P}_{\Delta}\left({ }^{n} E\right),\|\cdot\|_{\Delta}\right)$ is a quasi-normed space of $n$-homogeneous polynomials defined on $E$ such that the inclusion $\mathcal{P}_{\Delta}\left({ }^{n} E\right) \hookrightarrow \mathcal{P}\left({ }^{n} E\right)$ is continuous and $\mathcal{P}_{f}\left({ }^{n} E\right) \subset \mathcal{P}_{\Delta}\left({ }^{n} E\right)$. Let $C_{\Delta_{n}}>0$ be such that $\|P\| \leq C_{\Delta_{n}}\|P\|_{\Delta}$, for all $P \in \mathcal{P}_{\Delta}\left({ }^{n} E\right)$. Suppose that the normed space $\left(\mathcal{P}_{\Delta^{\prime}}\left({ }^{n} E^{\prime}\right),\|\cdot\|_{\Delta^{\prime}}\right) \subset \mathcal{P}\left({ }^{n} E^{\prime}\right)$ is such that the Borel transform

$$
\mathcal{B}:\left(\mathcal{P}_{\Delta}\left({ }^{n} E\right)^{\prime},\|\cdot\|\right) \rightarrow\left(\mathcal{P}_{\Delta^{\prime}}\left({ }^{n} E^{\prime}\right),\|\cdot\|_{\Delta^{\prime}}\right)
$$

given by $\mathcal{B}(T)(\varphi)=T\left(\varphi^{n}\right)$, for all $\varphi \in E^{\prime}$ and $T \in \mathcal{P}_{\Delta}\left({ }^{n} E\right)^{\prime}$, is a topological isomorphism.

We will show that the pair

$$
\left(\mathcal{P}_{\Delta}\left({ }^{n} E\right), \mathcal{P}_{\Delta^{\prime}}\left({ }^{n} E^{\prime}\right)\right)
$$

is a dual system. More precisely, we will prove that there exists a bilinear form $\langle\cdot ; \cdot\rangle$ on

$$
\mathcal{P}_{\Delta}\left({ }^{n} E\right) \times \mathcal{P}_{\Delta^{\prime}}\left({ }^{n} E^{\prime}\right)
$$

such that the following conditions hold:

(S1) $\langle P ; Q\rangle=0$ for all $Q \in \mathcal{P}_{\Delta^{\prime}}\left({ }^{n} E^{\prime}\right)$ implies $P=0$.

(S2) $\langle P ; Q\rangle=0$ for all $P \in \mathcal{P}_{\Delta}\left({ }^{n} E\right)$ implies $Q=0$.

Let

be defined by

$$
\langle\cdot, \cdot\rangle: \mathcal{P}_{\Delta}\left({ }^{n} E\right) \times \mathcal{P}_{\Delta^{\prime}}\left({ }^{n} E^{\prime}\right) \longrightarrow \mathbb{K}
$$

It is clear that $\langle\cdot, \cdot\rangle$ is bilinear.

$$
\langle P ; Q\rangle=\mathcal{B}^{-1}(Q)(P) .
$$


For $0 \neq Q \in \mathcal{P}_{\Delta^{\prime}}\left({ }^{n} E^{\prime}\right)$, we have $\mathcal{B}^{-1}(Q) \neq 0$ (because $\mathcal{B}$ is an isomorphism). Hence

$$
\langle P ; Q\rangle=\mathcal{B}^{-1}(Q)(P) \neq 0
$$

for some $P \in \mathcal{P}_{\Delta}\left({ }^{n} E\right)$, and so $(S 2)$ holds.

Now, if $0 \neq P \in \mathcal{P}_{\Delta}\left({ }^{n} E\right)$, then there is $x \in E$ such that $P(x) \neq 0$. We consider $A_{x} \in E^{\prime \prime}$ defined by

$$
A_{x}(\varphi)=\varphi(x),
$$

for all $\varphi \in E^{\prime}$ and define

$$
\begin{gathered}
T: \mathcal{P}_{\Delta}\left({ }^{n} E\right) \rightarrow \mathbb{K} \\
T(P)=P(x) .
\end{gathered}
$$

Obviously $T$ is linear. Moreover, $T$ is continuous and $\|T\| \leq C_{\Delta_{n}}\|x\|^{n}$. In fact, for every $P \in \mathcal{P}_{\Delta}\left({ }^{n} E\right)$

$$
|T(P)|=|P(x)| \leq\|P\|\|x\|^{n} \leq C_{\Delta_{n}}\|x\|^{n}\|P\|_{\Delta}
$$

and so $\|T\| \leq C_{\Delta_{n}}\|x\|^{n}$. Note that

$$
\mathcal{B}(T)(\varphi)=T\left(\varphi^{n}\right)=\varphi(x)^{n}=\left(A_{x}(\varphi)\right)^{n}
$$

for all $\varphi \in E^{\prime}$. We conclude that the polynomial

$$
\begin{gathered}
\left(A_{x}\right)^{n}: E^{\prime} \rightarrow \mathbb{K} \\
\left(A_{x}\right)^{n}(\varphi)=\varphi(x)^{n}
\end{gathered}
$$

belongs to $\mathcal{P}_{\Delta^{\prime}}\left({ }^{n} E^{\prime}\right)$ and

$$
<P,\left(A_{x}\right)^{n}>=\mathcal{B}^{-1}\left(\left(A_{x}\right)^{n}\right)(P)=T(P)=P(x) \neq 0 .
$$

Thus $(S 1)$ is proved and hence the pair $\left(\mathcal{P}_{\Delta}\left({ }^{n} E\right), \mathcal{P}_{\Delta^{\prime}}\left({ }^{n} E^{\prime}\right)\right)$ is a dual system.

Now, let

$$
U=\left\{P \in \mathcal{P}_{\Delta}\left({ }^{n} E\right) ;\|P\|_{\Delta} \leq 1\right\} .
$$

Since the bipolar of $U$, denoted by $U^{\circ \circ}$, is absorbing we can consider the corresponding gauge

$$
p_{U^{\circ \circ}}(P)=\inf \left\{\delta>0 ; P \in \delta U^{\circ \circ}\right\},
$$

defined for all $P$ in $\mathcal{P}_{\Delta}\left({ }^{n} E\right)$. Recall that the polar of $U$ is defined by

$$
U^{\circ}=\left\{Q \in \mathcal{P}_{\Delta^{\prime}}\left({ }^{n} E^{\prime}\right) ;|<P, Q>| \leq 1 \text { for all } P \in U\right\} .
$$

Hence

$$
\begin{aligned}
U^{\circ} & =\left\{Q \in \mathcal{P}_{\Delta^{\prime}}\left({ }^{n} E^{\prime}\right) ;\left|\mathcal{B}^{-1}(Q)(P)\right| \leq 1 \text { for all } P \in \mathcal{P}_{\Delta}\left({ }^{n} E\right),\|P\|_{\Delta} \leq 1\right\} \\
& =\left\{Q \in \mathcal{P}_{\Delta^{\prime}}\left({ }^{n} E^{\prime}\right) ;\left\|\mathcal{B}^{-1}(Q)\right\| \leq 1\right\} .
\end{aligned}
$$

Moreover,

$$
\begin{aligned}
U^{\circ \circ} & =\left\{P \in \mathcal{P}_{\Delta}\left({ }^{n} E\right) ;|<P, Q>| \leq 1 \text { for all } Q \in U^{\circ}\right\} \\
& =\left\{P \in \mathcal{P}_{\Delta}\left({ }^{n} E\right) ;\left|\mathcal{B}^{-1}(Q)(P)\right| \leq 1, \text { for all } Q \in \mathcal{P}_{\Delta^{\prime}}\left({ }^{n} E^{\prime}\right) \text { with }\left\|\mathcal{B}^{-1}(Q)\right\| \leq 1\right\}
\end{aligned}
$$

and hence

$$
p_{U^{\circ \circ}}(P)=\inf \left\{\delta>0 ;\left|\mathcal{B}^{-1}(Q)(P)\right| \leq \delta, \text { for all } Q \in \mathcal{P}_{\Delta^{\prime}}\left({ }^{n} E^{\prime}\right) \text { with }\left\|\mathcal{B}^{-1}(Q)\right\| \leq 1\right\} .
$$

Since

$$
\left|\mathcal{B}^{-1}(Q)(P)\right| \leq\left\|\mathcal{B}^{-1}(Q)\right\|\|P\|_{\Delta} \leq\|P\|_{\Delta},
$$

for all $Q \in \mathcal{P}_{\Delta^{\prime}}\left({ }^{n} E^{\prime}\right)$ with $\left\|\mathcal{B}^{-1}(Q)\right\| \leq 1$, it follows that

$$
p_{U^{\circ \circ}}(P) \leq\|P\|_{\Delta},
$$

for all $P \in \mathcal{P}_{\Delta}\left({ }^{n} E\right)$. 
Note that $p_{U^{\circ \circ}}$ is a norm on $\mathcal{P}_{\Delta}\left({ }^{n} E\right)$. In fact, we only have to prove that $p_{U^{\circ \circ}}(P)=0$ implies $P=0$. If $p_{U \circ 0}(P)=0$, then

$$
\left|\mathcal{B}^{-1}(Q)(P)\right|=0
$$

for all $Q \in \mathcal{P}_{\Delta^{\prime}}\left({ }^{n} E^{\prime}\right)$ with $\left\|\mathcal{B}^{-1}(Q)\right\| \leq 1$. So, we conclude that

$$
<P, Q>=\left|\mathcal{B}^{-1}(Q)(P)\right|=0
$$

for all $Q \in \mathcal{P}_{\Delta^{\prime}}\left({ }^{n} E^{\prime}\right)$. Hence, from $(S 1)$ it follows that $P=0$.

From now on we will often use the Bipolar Theorem, which asserts that the bipolar of $U$ coincides with the $\sigma\left(\mathcal{P}_{\Delta}\left({ }^{n} E\right), \mathcal{P}_{\Delta^{\prime}}\left({ }^{n} E^{\prime}\right)\right)$-closure of the absolutely convex hull $\Gamma(U)$ of $U$.

Proposition 2.1. If $P \in \mathcal{P}_{\Delta}\left({ }^{n} E\right)$ then

$$
\|P\| \leq C_{\Delta_{n}} p_{U^{\circ \circ}}(P) .
$$

Proof. We know that

$$
\|P\| \leq C_{\Delta_{n}}\|P\|_{\Delta}
$$

for all $P \in \mathcal{P}_{\Delta}\left({ }^{n} E\right)$. If $P$ belongs to the absolutely convex hull $\Gamma(U)$ of $U$, then

$$
P=\sum_{j=1}^{m} \lambda_{j} P_{j}
$$

where $P_{j} \in U, \lambda_{j} \in \mathbb{K}, j=1, \ldots, m$, for some $m \in \mathbb{N}$, and

$$
\sum_{j=1}^{m}\left|\lambda_{j}\right| \leq 1
$$

Since $P_{j} \in U$, we have

$$
\left\|P_{j}\right\| \leq C_{\Delta_{n}}\left\|P_{j}\right\|_{\Delta} \leq C_{\Delta_{n}}
$$

Therefore,

$$
\|P\|=\left\|\sum_{j=1}^{m} \lambda_{j} P_{j}\right\| \leq \sum_{j=1}^{m}\left|\lambda_{j}\right|\left\|P_{j}\right\| \leq C_{\Delta_{n}} \sum_{j=1}^{m}\left|\lambda_{j}\right| \leq C_{\Delta_{n}}
$$

for every $P \in \Gamma(U)$. Now if $P \in U^{\circ 0}$, which is the $\sigma\left(\left(\mathcal{P}_{\Delta}\left({ }^{n} E\right), \mathcal{P}_{\Delta^{\prime}}\left({ }^{n} E^{\prime}\right)\right)\right)$-closure of $\Gamma(U)$, let $\left(P_{i}\right)_{i \in I}$ be a net in $\Gamma(U)$ such that

$$
\lim _{i \in I}\left|<P_{i}, Q>\right|=|<P, Q>|
$$

for all $Q \in \mathcal{P}_{\Delta^{\prime}}\left({ }^{n} E^{\prime}\right)$. So we have

$$
\lim _{i \in I}\left|\mathcal{B}^{-1}(Q)\left(P_{i}\right)\right|=\left|\mathcal{B}^{-1}(Q)(P)\right|
$$

for all $Q \in \mathcal{P}_{\Delta^{\prime}}\left({ }^{n} E^{\prime}\right)$. In particular, for $x \in B_{E}$, we have

$$
\begin{aligned}
|P(x)| & =\left|\mathcal{B}^{-1}\left(\left(A_{x}\right)^{n}\right)(P)\right| \\
& =\lim _{i \in I}\left|\mathcal{B}^{-1}\left(\left(A_{x}\right)^{n}\right)\left(P_{i}\right)\right|=\lim _{i \in I}\left|P_{i}(x)\right| \stackrel{(2.2)}{\leq} C_{\Delta_{n}}\|x\| .
\end{aligned}
$$

Hence

$$
\|P\| \leq C_{\Delta_{n}}
$$

for every $P \in U^{\circ \circ}$. Finally, for $0 \neq P \in \mathcal{P}_{\Delta}\left({ }^{n} E\right)$, let

$$
R=\left(p_{U} \circ(P)\right)^{-1} P .
$$

We thus have

$$
p_{U} \circ(R)=1,
$$


and this implies that

$$
\left|\mathcal{B}^{-1}(Q)(R)\right| \leq 1
$$

for all $Q \in \mathcal{P}_{\Delta^{\prime}}\left({ }^{n} E^{\prime}\right)$ with $\left\|\mathcal{B}^{-1}(Q)\right\| \leq 1$. Thus $R \in U^{\circ \circ}$ and, consequently, from (2.3) we conclude that $\|R\| \leq C_{\Delta_{n}}$, i.e.,

and the result follows.

$$
\left\|\left(p_{U^{\circ \circ}}(P)\right)^{-1} P\right\| \leq C_{\Delta_{n}}
$$

We denote the completion of the space $\left(\mathcal{P}_{\Delta}\left({ }^{n} E\right), p_{U^{\circ}}\right)$ by $\left(\mathcal{P}_{\widetilde{\Delta}}\left({ }^{n} E\right),\|\cdot\|_{\widetilde{\Delta}}\right)$. So the restriction of $\|\cdot\|_{\widetilde{\Delta}}$ to $\mathcal{P}_{\Delta}\left({ }^{n} E\right)$ is $p_{U^{\circ \circ}}$ and Proposition 2.1] implies that

$$
\mathcal{P}_{\widetilde{\Delta}}\left({ }^{n} E\right) \subset \mathcal{P}\left({ }^{n} E\right)
$$

and

$$
\|P\| \leq C_{\Delta_{n}}\|P\|_{\widetilde{\Delta}}
$$

for all $P$ in $\mathcal{P}_{\widetilde{\Delta}}\left({ }^{n} E\right)$.

Definition 2.2. The elements of $\mathcal{P}_{\widetilde{\Delta}}\left({ }^{n} E\right)$ are called quasi- $\Delta n$-homogeneous polynomials.

Remark 2.3. When $\mathcal{P}_{\Delta}\left({ }^{n} E\right)$ is a Banach space then we have $\|\cdot\|_{\widetilde{\Delta}}=\|\cdot\|_{\Delta}$ and $\mathcal{P}_{\widetilde{\Delta}}\left({ }^{n} E\right)=\mathcal{P}_{\Delta}\left({ }^{n} E\right)$.

In fact, in this case, $U$ is the closed unit ball in $\mathcal{P}_{\Delta}\left({ }^{n} E\right)$, hence balanced and convex and $\Gamma(U)=U$. By using the Bipolar Theorem we have

$$
U^{\circ \circ}=\overline{\Gamma(U)}^{\sigma\left(\mathcal{P}_{\Delta}\left({ }^{n} E\right), \mathcal{P}_{\Delta^{\prime}}\left({ }^{n} E^{\prime}\right)\right)}=\bar{U}^{\sigma\left(\mathcal{P}_{\Delta}\left({ }^{n} E\right), \mathcal{P}_{\Delta^{\prime}}\left({ }^{n} E^{\prime}\right)\right)}=U,
$$

and the last equality follows from Banach-Mazur Theorem. Hence

$$
p_{U^{\circ \circ}}(P)=\inf \left\{\delta>0 ; P \in \delta U^{\circ \circ}\right\}=\inf \{\delta>0 ; P \in \delta U\}=\inf \left\{\delta>0 ;\|P\|_{\Delta} \leq \delta\right\}=\|P\|_{\Delta} .
$$

The next theorem plays a fundamental role in this work. It assures that the duals of $\mathcal{P}_{\widetilde{\Delta}}\left({ }^{n} E\right)$ and $\mathcal{P}_{\Delta}\left({ }^{n} E\right)$ are identified by the Borel transform.

Theorem 2.4. The linear mapping

$$
\begin{aligned}
\widetilde{\mathcal{B}}:\left(\mathcal{P}_{\widetilde{\Delta}}\left({ }^{n} E\right)^{\prime},\|\cdot\|\right) & \longrightarrow\left(\mathcal{P}_{\Delta^{\prime}}\left({ }^{n} E^{\prime}\right),\|\cdot\|_{\Delta^{\prime}}\right) \\
\widetilde{\mathcal{B}}(T)(\varphi) & =T\left(\varphi^{n}\right)
\end{aligned}
$$

is a topological isomorphism.

Proof. We know that $\left(\mathcal{P}_{\Delta}\left({ }^{n} E\right), p_{U^{\circ}}\right)$ is dense in $\left(\mathcal{P}_{\widetilde{\Delta}}\left({ }^{n} E\right),\|\cdot\|_{\widetilde{\Delta}}\right)$. Thus the topological duals of both spaces are isometrically isomorphic. So we only need to prove that $\mathcal{P}_{\Delta}\left({ }^{n} E\right)$ has the same topological dual for the norm $p_{U^{\circ} \circ}$ and for the quasi-norm $\|\cdot\|_{\Delta}$. By (2.1), for each $T \in\left(\mathcal{P}_{\Delta}\left({ }^{n} E\right), p_{U^{\circ \circ}}\right)^{\prime}$ we have

$$
\sup _{P \in U}|T(P)| \leq \sup _{p_{U} \circ \circ(P) \leq 1}|T(P)|
$$

and this implies that the inclusion

$$
\left(\mathcal{P}_{\Delta}\left({ }^{n} E\right), p_{U^{\circ 0}}\right)^{\prime} \hookrightarrow\left(\mathcal{P}_{\Delta}\left({ }^{n} E\right),\|\cdot\|_{\Delta}\right)^{\prime}
$$

is continuous. Now, let $T \in\left(\mathcal{P}_{\Delta}\left({ }^{n} E\right),\|\cdot\|_{\Delta}\right)^{\prime}$. If $P \in \Gamma(U)$, then

$$
P=\sum_{j=1}^{m} \lambda_{j} P_{j},
$$

where $P_{j} \in U, \lambda_{j} \in \mathbb{K}, j=1, \ldots, m$, for some $m \in \mathbb{N}$, and

$$
\sum_{j=1}^{m}\left|\lambda_{j}\right| \leq 1 .
$$


Thus

$$
|T(P)| \leq \sum_{j=1}^{m}\left|\lambda_{j}\right|\left\|T\left(P_{j}\right)\right\| \leq \sup _{Q \in U}|T(Q)| \sum_{j=1}^{m}\left|\lambda_{j}\right| \leq \sup _{Q \in U}|T(Q)|<+\infty .
$$

If $P \in U^{\circ \circ}$, then there exists a net $\left(P_{i}\right)_{i \in I} \subset \Gamma(U)$ such that

$$
|T(P)|=\lim _{i \in I}\left|T\left(P_{i}\right)\right| \stackrel{\sqrt[2.5]{\leq}}{\leq} \sup _{Q \in U}|T(Q)|<+\infty .
$$

Hence $T$ is bounded over $U^{\circ \circ}$ and so continuous for $p_{U^{\circ \circ}}$, as we wanted to show.

The next result will be necessary in Section [4. It is clear that since $\mathcal{P}_{f}\left({ }^{n} E\right)$ is contained in $\mathcal{P}_{\Delta}\left({ }^{n} E\right)$, then $\mathcal{P}_{f}\left({ }^{n} E\right)$ is contained in $\mathcal{P}_{\widetilde{\Delta}}\left({ }^{n} E\right)$.

Proposition 2.5. Let $n \in \mathbb{N}$.

(a) If there exists $K>0$ such that $\left\|\varphi^{n}\right\|_{\Delta} \leq K\|\varphi\|^{n}$, for all $\varphi \in E^{\prime}$, then

$$
\left\|\varphi^{n}\right\|_{\widetilde{\Delta}} \leq K\|\varphi\|^{n} \leq K C_{\Delta_{1}}^{n}\|\varphi\|_{\widetilde{\Delta}}^{n}
$$

for all $\varphi \in E^{\prime}$.

(b) If $\mathcal{P}_{f}\left({ }^{n} E\right)$ is dense in $\left(\mathcal{P}_{\Delta}\left({ }^{n} E\right),\|\cdot\|_{\Delta}\right)$, then $\mathcal{P}_{f}\left({ }^{n} E\right)$ is dense in $\left(\mathcal{P}_{\widetilde{\Delta}}\left({ }^{n} E\right),\|\cdot\|_{\widetilde{\Delta}}\right)$.

Proof. (a) By inequality (2.1) we have $\|P\|_{\widetilde{\Delta}} \leq\|P\|_{\Delta}$, for every $P \in \mathcal{P}_{\Delta}\left({ }^{n} E\right)$. In particular, for every $\varphi \in E^{\prime}$

Besides, 2.4) assures that

$$
\left\|\varphi^{n}\right\|_{\widetilde{\Delta}} \leq\left\|\varphi^{n}\right\|_{\Delta} \leq K\|\varphi\|^{n}
$$

$$
\|\varphi\| \leq C_{\Delta_{1}}\|\varphi\|_{\widetilde{\Delta}}
$$

for every $\varphi \in E^{\prime}$. Now the result follows from the last two inequalities.

(b) We know that $p_{U^{\circ \circ}}(\cdot) \leq\|\cdot\|_{\Delta}$ (see (2.1)) and $p_{U^{\circ \circ}}(P)=\|P\|_{\widetilde{\Delta}}$, for all $P \in \mathcal{P}_{\Delta}\left({ }^{n} E\right)$. Using this fact and the density of $\mathcal{P}_{f}\left({ }^{n} E\right)$ in $\left(\mathcal{P}_{\Delta}\left({ }^{n} E\right),\|\cdot\|_{\Delta}\right)$, the result follows.

Now we are interested in connecting the previous construction with the concept of holomorphy type that we recall below. The notation for the derivatives of polynomials that we use are the same introduced by L. Nachbin in [51].

Definition 2.6. A holomorphy type $\Theta$ from $E$ to $F$ is a sequence of Banach spaces $\left(\mathcal{P}_{\Theta}\left({ }^{n} E ; F\right)\right)_{n=0}^{\infty}$, the norm on each of them being denoted by $\|\cdot\|_{\Theta}$, such that the following conditions hold true:

(1) Each $\mathcal{P}_{\Theta}\left({ }^{n} E ; F\right)$ is a linear subspace of $\mathcal{P}\left({ }^{n} E ; F\right)$.

(2) $\mathcal{P}_{\Theta}\left({ }^{0} E ; F\right)$ coincides with $\mathcal{P}\left({ }^{0} E ; F\right)=F$ as a normed vector space.

(3) There is a real number $\sigma \geq 1$ for which the following is true: given any $k \in \mathbb{N}_{0}, n \in \mathbb{N}_{0}, k \leq n$, $a \in E$ and $P \in \mathcal{P}_{\Theta}\left({ }^{n} E ; F\right)$, we have

$$
\begin{gathered}
\hat{d}^{k} P(a) \in \mathcal{P}_{\Theta}\left({ }^{k} E ; F\right) \text { and } \\
\left\|\frac{1}{k !} \hat{d}^{k} P(a)\right\|_{\Theta} \leq \sigma^{n}\|P\|_{\Theta}\|a\|^{n-k} .
\end{gathered}
$$

It is plain that each inclusion $\mathcal{P}_{\Theta}\left({ }^{n} E ; F\right) \subseteq \mathcal{P}\left({ }^{n} E ; F\right)$ is continuous and that $\|P\| \leq \sigma^{n}\|P\|_{\Theta}$ for every $P \in \mathcal{P}_{\Theta}\left({ }^{n} E ; F\right)$.

The definition of holomorphy type motivates the next definition for quasi-normed spaces of homogeneous polynomials. 
Definition 2.7. For each $n \in \mathbb{N}_{0}$, let $\left(\mathcal{P}_{\Delta}\left({ }^{n} E\right),\|\cdot\|_{\Delta}\right)$ be a quasi-normed space, where $\mathcal{P}_{\Delta}\left({ }^{0} E\right)=\mathbb{C}$. The sequence $\left(\mathcal{P}_{\Delta}\left({ }^{n} E\right)\right)_{n=0}^{\infty}$ is stable for derivatives if

(1) $\hat{d}^{k} P(x) \in \mathcal{P}_{\Delta}\left({ }^{k} E\right)$ for each $n \in \mathbb{N}_{0}, P \in \mathcal{P}_{\Delta}\left({ }^{n} E\right), k=0,1, \ldots, n$ and $x \in E$.

(2) For each $n \in \mathbb{N}_{0}, k=0,1, \ldots, n$, there is a constant $C_{n, k} \geq 0$ such that

$$
\left\|\hat{d}^{k} P(x)\right\|_{\Delta} \leq C_{n, k}\|P\|_{\Delta}\|x\|^{n-k},
$$

for all $x \in E$.

Theorem 2.8. Let $\left(\mathcal{P}_{\Delta}\left({ }^{n} E\right)\right)_{n=0}^{\infty}$ be a sequence stable for derivatives. If $P \in \mathcal{P}_{\widetilde{\Delta}}\left({ }^{n} E\right)$, then

$$
\hat{d}^{k} P(x) \in \mathcal{P}_{\widetilde{\Delta}}\left({ }^{k} E\right)
$$

and

$$
\left\|\hat{d}^{k} P(x)\right\|_{\widetilde{\Delta}} \leq C_{n, k}\|P\|_{\widetilde{\Delta}}\|x\|^{n-k},
$$

for every $k=0,1, \ldots, n$ and $x \in E$, where $C_{n, k}$ is the constant of Definition 2.7.

Proof. By hypothesis we have

$$
\left\|\hat{d}^{k} P(x)\right\|_{\Delta} \leq C_{n, k}\|P\|_{\Delta}\|x\|^{n-k},
$$

for all $P \in \mathcal{P}_{\Delta}\left({ }^{n} E\right), k=0,1, \ldots, n$ and $x \in E$. Let $U_{k}=\left\{Q \in \mathcal{P}_{\Delta}\left({ }^{k} E\right) ;\|Q\|_{\Delta} \leq 1\right\}$ and let $V_{k}$ be the absolutely convex hull of $U_{k}$. Let $p_{V_{k}}$ be the gauge of $V_{k}$ (note that $p_{V_{k}}$ is a norm, since $V_{k}$ is a bounded, balanced and convex neighborhood of zero). Consider

$$
\begin{gathered}
\psi: \mathcal{P}_{\Delta}\left({ }^{n} E\right) \rightarrow \mathcal{P}_{\Delta}\left({ }^{k} E\right) \\
\psi(P)=\hat{d}^{k} P(x) .
\end{gathered}
$$

We know that

$$
p_{V_{k}}(Q) \leq\|Q\|_{\Delta}
$$

for every $Q \in \mathcal{P}_{\Delta}\left({ }^{k} E\right)$. In fact, since $V_{k}$ is convex, balanced and absorbing, we have

$$
V_{k} \subset\left\{Q \in \mathcal{P}_{\Delta}\left({ }^{k} E\right) ; p_{V_{k}}(Q) \leq 1\right\}
$$

and, for $Q \in \mathcal{P}_{\Delta}\left({ }^{k} E\right), Q \neq 0$, we have

Hence,

$$
\left\|\frac{Q}{\|Q\|_{\Delta}}\right\|_{\Delta}=1
$$

and

$$
\frac{Q}{\|Q\|_{\Delta}} \in U_{k} \subset V_{k}
$$

which shows (2.7). From (2.7) we get

$$
p_{V_{k}}\left(\frac{Q}{\|Q\|_{\Delta}}\right) \leq 1
$$

$$
p_{V_{k}}\left(\hat{d}^{k} P(x)\right) \leq\left\|\hat{d}^{k} P(x)\right\|_{\Delta} \stackrel{\sqrt{2.6})}{\leq} C_{n, k}\|P\|_{\Delta}\|x\|^{n-k}
$$

for every $P \in \mathcal{P}_{\Delta}\left({ }^{n} E\right)$. Now let $Q \in V_{n}$. Then

$$
Q=\sum_{j=1}^{m} \lambda_{j} P_{j}
$$

with $P_{j} \in U_{n}, j=1, \ldots, m$ and $\left|\lambda_{1}\right|+\cdots+\left|\lambda_{m}\right|=1$. Hence

$$
p_{V_{k}}\left(\hat{d}^{k} Q(x)\right) \leq \sum_{j=1}^{m}\left|\lambda_{j}\right| p_{V_{k}}\left(\hat{d}^{k} P_{j}(x)\right)
$$




$$
\begin{aligned}
& \leq C_{n, k}\left\|P_{j}\right\|_{\Delta}\|x\|^{n-k} \\
& \leq C_{n, k}\|x\|^{n-k} .
\end{aligned}
$$

for every $Q \in V_{n}$.

If $P \in \mathcal{P}_{\Delta}\left({ }^{n} E\right), P \neq 0$, then for every $\varepsilon>0$ we have

$$
p_{V_{n}}\left(\frac{P}{p_{V_{n}}(P)+\varepsilon}\right)<1
$$

and it follows from the definition of $p_{V_{n}}$ that

$$
\frac{P}{p_{V_{n}}(P)+\varepsilon} \in 1 V_{n}=V_{n} .
$$

Hence, from (2.9) we have

$$
p_{V_{k}}\left(\hat{d}^{k}\left(\frac{P}{p_{V_{n}}(P)+\varepsilon}\right)(x)\right) \leq C_{n, k}\|x\|^{n-k}
$$

for every $\varepsilon>0$. Since $\varepsilon>0$ is arbitrary, we obtain

$$
p_{V_{k}}\left(\hat{d}^{k} P(x)\right) \leq C_{n, k}\|x\|^{n-k} p_{V_{n}}(P) .
$$

From the Bipolar Theorem we know that $U_{n}^{\circ \circ}$ is the weak closure of $V_{n}$. It is clear that (2.8) holds for $n$ in the place of $k$, so we also have

$$
V_{n} \subset\left\{Q \in \mathcal{P}_{\Delta}\left({ }^{n} E\right) ; p_{V_{n}}(Q) \leq 1\right\} .
$$

Note that $\left(\mathcal{P}_{\Delta}\left({ }^{n} E\right), p_{V_{n}}\right)$ is consistent with the dual system $\left(\mathcal{P}_{\Delta}\left({ }^{n} E\right), \mathcal{P}_{\Delta^{\prime}}\left({ }^{n} E^{\prime}\right)\right)$, and this means that the dual of $\mathcal{P}_{\Delta}\left({ }^{n} E\right)$ endowed with the topologies $p_{V_{n}}$ and $\|\cdot\|_{\Delta}$ is the same. In fact,

$$
p_{U_{n}^{\circ \circ}} \leq p_{V_{n}} \leq p_{U_{n}} \leq\|\cdot\|_{\Delta}
$$

and $\left(\mathcal{P}_{\Delta}\left({ }^{n} E\right), p_{U_{n}^{\circ \circ}}\right)$ is dense in $\left(\mathcal{P}_{\widetilde{\Delta}}\left({ }^{n} E\right),\|\cdot\|_{\tilde{\Delta}}\right)$. Hence

$$
\begin{aligned}
\left(\mathcal{P}_{\Delta}\left({ }^{n} E\right), p_{U_{n}^{\circ}}\right)^{\prime} & =\left(\mathcal{P}_{\widetilde{\Delta}}\left({ }^{n} E\right),\|\cdot\|_{\widetilde{\Delta}}\right)^{\prime} \\
& =\left(\mathcal{P}_{\Delta^{\prime}}\left({ }^{n} E^{\prime}\right),\|\cdot\|_{\Delta^{\prime}}\right) \\
& =\left(\mathcal{P}_{\Delta}\left({ }^{n} E\right),\|\cdot\|_{\Delta}\right)^{\prime} .
\end{aligned}
$$

From [55, 3.1 p. 130] we know that the closure of a convex set is the same no matter how we choose the topology (consistent with the dual system). Since $V_{n}$ is convex, we have

$$
\begin{aligned}
\left\{Q \in \mathcal{P}_{\Delta}\left({ }^{n} E\right) ; p_{V_{n}}(Q) \leq 1\right\} & ={\overline{\left\{Q \in \mathcal{P}_{\Delta}\left({ }^{n} E\right) ; p_{V_{n}}(Q) \leq 1\right\}}}^{p_{V_{n}}} \\
& ={\overline{\left\{Q \in \mathcal{P}_{\Delta}\left({ }^{n} E\right) ; p_{V_{n}}(Q) \leq 1\right\}}}^{\sigma\left(\mathcal{P}_{\Delta}\left({ }^{n} E\right), \mathcal{P}_{\Delta^{\prime}}\left({ }^{n} E^{\prime}\right)\right)} .
\end{aligned}
$$

Hence

$$
U_{n}^{\circ \circ}={\overline{V_{n}}}^{\sigma\left(\mathcal{P}_{\Delta}\left({ }^{n} E\right), \mathcal{P}_{\Delta^{\prime}}\left({ }^{n} E^{\prime}\right)\right)}=\left\{Q \in \mathcal{P}_{\Delta}\left({ }^{n} E\right) ; p_{V_{n}}(Q) \leq 1\right\}
$$

Thus we have

$$
\begin{aligned}
p_{U_{k}^{\circ \circ}}(\psi(P)) & \leq p_{V_{k}}(\psi(P)) \\
& \stackrel{2.10}{\leq} C_{n, k}\|x\|^{n-k} p_{V_{n}}(P) \\
& \stackrel{2.11}{\leq} C_{n, k}\|x\|^{n-k}
\end{aligned}
$$

for every $P \in U_{n}^{\circ \circ}$.

Now, let $P \in \mathcal{P}_{\Delta}\left({ }^{n} E\right), P \neq 0$. From the argument used just after (2.3) we have

$$
\frac{P}{p_{U_{n}^{\circ \circ}}(P)} \in U_{n}^{\circ \circ}
$$


and hence

$$
p_{U_{k}^{\circ \circ}}\left(\hat{d}^{k}\left(\frac{P}{p_{U_{n}^{\circ} \circ}(P)}\right)(x)\right) \stackrel{\sqrt[\mid 2.12]]{\leq}}{\leq} C_{n, k}\|x\|^{n-k}
$$

and we finally conclude that

$$
p_{U_{k}^{\circ \circ}}(\psi(P)) \leq C_{n, k}\|x\|^{n-k} p_{U_{n}^{\circ \circ}}(P) .
$$

This implies that $\psi$ is a continuous linear mapping from $\left(\mathcal{P}_{\Delta}\left({ }^{n} E\right), p_{U_{n}^{\circ \circ}}\right)$ into $\left(\mathcal{P}_{\Delta}\left({ }^{k} E\right), p_{U_{k}^{\circ \circ}}\right)$. We can now extend $\psi$ to the completions $\left(\mathcal{P}_{\Delta}\left({ }^{n} E\right),\|\cdot\|_{\widetilde{\Delta}}\right)$ and $\left(\mathcal{P}_{\Delta}\left({ }^{k} E\right),\|\cdot\|_{\widetilde{\Delta}}\right)$ and the proof is done.

Corollary 2.9. If $\left(\mathcal{P}_{\Delta}\left({ }^{n} E\right)\right)_{n=0}^{\infty}$ is stable for derivatives with $C_{n, k} \leq \frac{n !}{(n-k) !}$, then $\left(\mathcal{P}_{\widetilde{\Delta}}\left({ }^{n} E\right)\right)_{n=0}^{\infty}$ is a holomorphy type.

Proof. Since conditions (1) and (2) of Definition 2.6 are clear, we only have to prove (3). We will show that for $\sigma=2$, we obtain (3). Let $P \in \mathcal{P}_{\widetilde{\Delta}}\left({ }^{n} E\right), k \in \mathbb{N}_{0}, k \leq n$ and $x \in E$. By Theorem 2.8 we have $\hat{d}^{k} P(x) \in \mathcal{P}_{\widetilde{\Delta}}\left({ }^{k} E\right)$ and

Hence

$$
\left\|\hat{d}^{k} P(x)\right\|_{\widetilde{\Delta}} \leq \frac{n !}{(n-k) !}\|P\|_{\widetilde{\Delta}}\|x\|^{n-k}
$$

as we wanted to show.

$$
\left\|\frac{1}{k !} \hat{d}^{k} P(x)\right\|_{\widetilde{\Delta}} \leq \frac{n !}{k !(n-k) !}\|P\|_{\widetilde{\Delta}}\|x\|^{n-k} \leq 2^{n}\|P\|_{\widetilde{\Delta}}\|x\|^{n-k},
$$

Corollary 2.9 tells us how to use the results of this section to obtain a holomorphy type $\left(\mathcal{P}_{\widetilde{\Delta}}\left({ }^{n} E\right)\right)_{n=0}^{\infty}$ from $\left(\mathcal{P}_{\Delta}\left({ }^{n} E\right)\right)_{n=0}^{\infty}$. This result will be useful in Section 4

\section{Prerequisites For the applichtions}

We are interested in applying the results of the previous section to obtain new examples of hypercyclic convolution operators and new existence and approximation results for convolution equations. We start presenting a little of the state of the art of both topics.

If $X$ is a topological space, a map $f: X \rightarrow X$ is hypercyclic if the set $\left\{x, f(x), f^{2}(x), \ldots\right\}$ is dense in $X$ for some $x \in X$. In this case, $x$ is said to be a hypercyclic vector for $f$. Hypercyclic translation and differentiation operators on spaces of entire functions of one complex variable were first investigated by Birkhoff [7] and MacLane [38. Godefroy and Shapiro [31 pushed these results quite further by proving that every convolution operator on spaces of entire functions of several complex variables which is not a scalar multiple of the identity is hypercyclic. For the theory of hypercyclic operators and its ramifications we refer to [2, 4, 32 and references therein. We remark that several results on the hypercyclicity of operators on spaces of entire functions on infinitely many complex variables appeared later (see, e.g., 3, 5, 6, 12, 13, 30, 32, 49, 52 ). In 2007, Carando, Dimant and Muro 12 proved some general results, including a solution to a problem posed in [1, that encompass as particular cases several of the above mentioned results. In [5], using the theory of holomorphy types, Bertoloto, Botelho, Fávaro and Jatobá generalized the results of [12 to a more general setting. For instance, the following theorem from [5, when restricted to $E=\mathbb{C}^{n}$ and $\mathcal{P}_{\Theta}\left({ }^{m} \mathbb{C}^{n}\right)=\mathcal{P}\left({ }^{m} \mathbb{C}^{n}\right)$ recovers the famous result of Godefroy and Shapiro 31 on the hypercyclicity of convolution operators on $\mathcal{H}\left(\mathbb{C}^{n}\right)$ :

Theorem [5, Theorem 2.7] Let $E^{\prime}$ be separable and $\left(\mathcal{P}_{\Theta}\left({ }^{m} E\right)\right)_{m=0}^{\infty}$ be a $\pi_{1}$-holomorphy type from $E$ to $\mathbb{C}$. Then every convolution operator on $\mathcal{H}_{\Theta b}(E)$ which is not a scalar multiple of the identity is hypercyclic.

However, the spaces $\mathcal{P}_{\Theta}\left({ }^{m} E\right)$ need to be Banach spaces and thus $\mathcal{H}_{\Theta b}(E)$ becomes a Fréchet space. When the spaces $\mathcal{P}_{\Theta}\left({ }^{m} E\right)$ are quasi-Banach, the respective space $\mathcal{H}_{\Theta b}(E)$ is not Fréchet and then some arguments used to prove the result above do not work.

Also, several spaces of holomorphic mappings, which have arisen with the development of the theory of polynomial and operator ideals (see, for instance [17, 53, 54]), are not Fréchet spaces and thus the investigation of this more general setting seems to be relevant. 
The same problem happens in the investigation of existence and approximation results for convolution equations. This line of investigation was initiated by Malgrange [39] and developed by several authors (see, for instance [14, 15, 16, 22, 23, 24, 25, 26, 27, 33, 34, 40, 41, 42, 43, 45, 46, 50,). In this context, a result of [26] (refined in [5]) gives a general method to prove existence and approximation results for convolution equations defined on certain spaces of entire functions of bounded type. The general process to prove existence and approximation results for convolution equations on $\mathcal{H}_{\Theta b}(E)$ involves three main steps:

(i) To establish an isomorphism between the topological dual of $\mathcal{H}_{\Theta b}(E)$ and a certain space $\mathcal{E}$ of exponential-type holomorphic functions via Borel transform.

(ii) To prove a division theorem for holomorphic functions on $\mathcal{E}$, that is, if $f g=h, g \neq 0, g, h \in \mathcal{E}$, $f \in \mathcal{H}\left(E^{\prime}\right)$, then it is possible to show that $f \in \mathcal{E}$.

(iii) To handle the results of (i) and (ii) and use Hahn-Banach type theorems and the DieudonnéSchwartz theorem that appears in 21].

The absence of Hahn-Banach and Dieudonné-Schwartz theorems for more general settings is a crucial obstacle for the development of a general theory. For some classes of polynomials $\mathcal{P}_{\Theta}\left({ }^{m} E\right)$ there are duality results via Borel transform but the step (iii) can not be accomplished.

The results of Section 1, together with the results of [5], allow us to deal with these problematic cases of hypercyclicity and existence and approximation results for convolution equations. We shall use the results of Section 1 in such way that steps (i)-(iii) are applicable. It is worth mentioning that the proof of division theorems (step (ii)) for holomorphic functions is always a hardwork (see, e.g., 15, 16, 25, 35, 37, 40, 44).

Now we present some preliminary results for the applications.

Definition 3.1. 26, Definition 2.2] Let $\left(\mathcal{P}_{\Theta}\left({ }^{m} E ; F\right)\right)_{m=0}^{\infty}$ be a holomorphy type from $E$ to $F$. A given $f \in \mathcal{H}(E ; F)$ is said to be of $\Theta$-holomorphy type of bounded type if

(i) $\hat{d}^{m} f(0) \in \mathcal{P}_{\Theta}\left({ }^{m} E ; F\right)$, for all $m \in \mathbb{N}_{0}$,

(ii) $\lim _{m \rightarrow \infty}\left(\frac{1}{m !}\left\|\hat{d}^{m} f(0)\right\|_{\Theta}\right)^{\frac{1}{m}}=0$.

The vector subspace of $\mathcal{H}(E ; F)$ of all such $f$ is denoted by $\mathcal{H}_{\Theta b}(E ; F)$ and becomes a Fréchet space with the topology $\tau_{\Theta}$ generated by the family of seminorms

$$
f \in \mathcal{H}_{\Theta b}(E ; F) \mapsto\|f\|_{\Theta, \rho}=\sum_{m=0}^{\infty} \frac{\rho^{m}}{m !}\left\|\hat{d}^{m} f(0)\right\|_{\Theta},
$$

for all $\rho>0$ (see [26, Proposition 2.3]).

When $F=\mathbb{C}$ we represent $\mathcal{H}_{\Theta b}(E ; \mathbb{C}):=\mathcal{H}_{\Theta b}(E)$.

The next two definitions are slight variations of the concepts of $\pi_{1}$ and $\pi_{2}$ holomorphy types (originally introduced in [26]) and they can be found in [5].

Definition 3.2. A holomorphy type $\left(\mathcal{P}_{\Theta}\left({ }^{m} E ; F\right)\right)_{m=0}^{\infty}$ from $E$ to $F$ is said to be a $\pi_{1}$-holomorphy type if the following conditions hold:

(i) Polynomials of finite type belong to $\left(\mathcal{P}_{\Theta}\left({ }^{m} E ; F\right)\right)_{m=0}^{\infty}$ and there exists $K>0$ such that

$$
\left\|\phi^{m} \cdot b\right\|_{\Theta} \leq K^{m}\|\phi\|^{m} \cdot\|b\|
$$

for all $\phi \in E^{\prime}, b \in F$ and $m \in \mathbb{N}$;

(ii) For each $m \in \mathbb{N}_{0}, \mathcal{P}_{f}\left({ }^{m} E ; F\right)$ is dense in $\left(\mathcal{P}_{\Theta}\left({ }^{m} E ; F\right),\|\cdot\|_{\Theta}\right)$.

Definition 3.3. A holomorphy type $\left(\mathcal{P}_{\Theta}\left({ }^{m} E\right)\right)_{m=0}^{\infty}$ from $E$ to $\mathbb{C}$ is said to be a $\pi_{2}$-holomorphy type if for each $T \in\left[\mathcal{H}_{\Theta b}(E)\right]^{\prime}, m \in \mathbb{N}_{0}$ and $k \in \mathbb{N}_{0}, k \leq m$, the following conditions hold:

(i) If $P \in \mathcal{P}_{\Theta}\left({ }^{m} E\right)$ and $A: E^{m} \longrightarrow \mathbb{C}$ is the unique continuous symmetric m-linear mapping such that $P=\hat{A}$, then the $(m-k)$-homogeneous polynomial

$$
T\left(\widehat{A(\cdot)^{k}}\right): E \longrightarrow \mathbb{C}
$$




$$
y \mapsto T\left(A(\cdot)^{k} y^{m-k}\right)
$$

belongs to $\mathcal{P}_{\Theta}\left({ }^{m-k} E\right)$;

(ii) For constants $C, \rho>0$ such that

$$
|T(f)| \leq C\|f\|_{\Theta, \rho} \text { for every } f \in \mathcal{H}_{\Theta b}(E)
$$

(which exist since $\left.T \in\left[\mathcal{H}_{\Theta b}(E)\right]^{\prime}\right)$, there is a constant $K>0$ such that

$$
\left\|T\left(\widehat{A(\cdot)^{k}}\right)\right\|_{\Theta} \leq C \cdot K^{m} \rho^{k}\|P\|_{\Theta} \text { for every } P \in \mathcal{P}_{\Theta}\left({ }^{m} E\right) \text {. }
$$

When we write " $\Theta$ is a $\pi_{1}-\pi_{2}$-holomorphy type", it means that $\Theta$ is a $\pi_{1}$ and a $\pi_{2}$-holomorphy type.

Let $\Theta$ be a $\pi_{1}$-holomorphy type from $E$ to $F$. It is clear that the Borel transform

$$
\mathcal{B}_{\Theta}:\left[\mathcal{P}_{\Theta}\left({ }^{m} E ; F\right)\right]^{\prime} \longrightarrow \mathcal{P}\left({ }^{m} E^{\prime} ; F^{\prime}\right), \mathcal{B}_{\Theta} T(\phi)(y)=T\left(\phi^{m} y\right),
$$

for $T \in\left[\mathcal{P}_{\Theta}\left({ }^{m} E ; F\right)\right]^{\prime}, \phi \in E^{\prime}$ and $y \in F$, is well-defined and linear. Moreover, by (i) and (ii) of Definition 3.2. $\mathcal{B}_{\Theta}$ is continuous and injective. So, denoting the range of $\mathcal{B}_{\Theta}$ in $\mathcal{P}\left({ }^{m} E^{\prime} ; F^{\prime}\right)$ by $\mathcal{P}_{\Theta^{\prime}}\left({ }^{m} E^{\prime} ; F^{\prime}\right)$, the correspondence

$$
\mathcal{B}_{\Theta} T \in \mathcal{P}_{\Theta^{\prime}}\left({ }^{m} E^{\prime} ; F^{\prime}\right) \mapsto\left\|\mathcal{B}_{\Theta} T\right\|_{\Theta^{\prime}}:=\|T\|
$$

defines a norm on $\mathcal{P}_{\Theta^{\prime}}\left({ }^{m} E^{\prime} ; F^{\prime}\right)$.

In this fashion the spaces $\left(\left[\mathcal{P}_{\Theta}\left({ }^{m} E ; F\right)\right]^{\prime},\|\cdot\|\right)$ and $\left(\mathcal{P}_{\Theta^{\prime}}\left({ }^{m} E^{\prime} ; F^{\prime}\right),\|\cdot\|_{\Theta^{\prime}}\right)$ are isometrically isomorphic. For more details on this isomorphism we refer [5] or [26].

Definition 3.4. 5, Definition 2.6] Let $\Theta$ be a holomorphy type from $E$ to $\mathbb{C}$.

(a) For $a \in E$ and $f \in \mathcal{H}_{\Theta b}(E)$, the translation of $f$ by $a$ is defined by

$$
\tau_{a} f: E \longrightarrow \mathbb{C},\left(\tau_{a} f\right)(x)=f(x-a) .
$$

$B y$ [26, Proposition 2.2] we have $\tau_{a} f \in \mathcal{H}_{\Theta b}(E)$.

(b) A continuous linear operator $L: \mathcal{H}_{\Theta b}(E) \longrightarrow \mathcal{H}_{\Theta b}(E)$ is called a convolution operator on $\mathcal{H}_{\Theta b}(E)$ if it is translation invariant, that is,

$$
L\left(\tau_{a} f\right)=\tau_{a}(L(f))
$$

for all $a \in E$ and $f \in \mathcal{H}_{\Theta b}(E)$.

(c) For each functional $T \in\left[\mathcal{H}_{\Theta b}(E)\right]^{\prime}$, the operator $\bar{\Gamma}_{\Theta}(T)$ is defined by

$$
\bar{\Gamma}_{\Theta}(T): \mathcal{H}_{\Theta b}(E) \longrightarrow \mathcal{H}_{\Theta b}(E), \bar{\Gamma}_{\Theta}(T)(f)=T * f,
$$

where the convolution product $T * f$ is defined by

$$
(T * f)(x)=T\left(\tau_{-x} f\right) \text { for every } x \in E .
$$

(d) $\delta_{0} \in\left[\mathcal{H}_{\Theta b}(E)\right]^{\prime}$ is the linear functional defined by

$$
\delta_{0}: \mathcal{H}_{\Theta b}(E) \longrightarrow \mathbb{C}, \delta_{0}(f)=f(0) .
$$

3.1. Hypercyclicity results. Using the techniques developed in Section 2 in the final section we shall provide new nontrivial applications of the following hypercyclicity results:

Theorem 3.5. [5. Theorem 2.7] Let $E^{\prime}$ be separable and $\left(\mathcal{P}_{\Theta}\left({ }^{m} E\right)\right)_{m=0}^{\infty}$ be a $\pi_{1}$-holomorphy type from $E$ to $\mathbb{C}$. Then every convolution operator on $\mathcal{H}_{\Theta b}(E)$ which is not a scalar multiple of the identity is hypercyclic.

Theorem 3.6. [5. Theorem 2.8] Let $E^{\prime}$ be separable, $\left(\mathcal{P}_{\Theta}\left({ }^{m} E\right)\right)_{m=0}^{\infty}$ be a $\pi_{1}-\pi_{2}$-holomorphy type and $T \in\left[\mathcal{H}_{\Theta b}(E)\right]^{\prime}$ be a linear functional which is not a scalar multiple of $\delta_{0}$. Then $\bar{\Gamma}_{\Theta}(T)$ is a convolution operator that is not a scalar multiple of the identity, hence hypercyclic. 
Remark 3.7. Since the proofs of Theorems 3.5 and 3.6 are based on the hypercyclicity criterion obtained by Kitai [36] and later on rediscovered by Gethner and Shapiro [30, the convolution operators of these theorems are in fact mixing, a property stronger than hypercyclicity.

\subsection{Existence and approximation results.}

Definition 3.8. Let $\left(\mathcal{P}_{\Theta}\left({ }^{m} E\right)\right)_{m=0}^{\infty}$ be a $\pi_{1}$-holomorphy type from $E$ to $\mathbb{C}$. An entire function $f \in \mathcal{H}\left(E^{\prime}\right)$ is said to be of $\Theta^{\prime}$-exponential type if

(i) $\hat{d}^{m} f(0) \in \mathcal{P}_{\Theta^{\prime}}\left({ }^{m} E^{\prime}\right)$ for every $m \in \mathbb{N}_{0}$;

(ii) There are constants $C \geq 0$ and $c>0$ such that

$$
\left\|\hat{d}^{m} f(0)\right\|_{\Theta^{\prime}} \leq C c^{m},
$$

for all $m \in \mathbb{N}_{0}$.

The vector space of all such functions is denoted by $\operatorname{Exp}_{\Theta^{\prime}}\left(E^{\prime}\right)$.

Definition 3.9. 26, Definition 4.1] Let $U$ be an open subset of $E$ and $\mathcal{F}(U)$ a collection of holomorphic functions from $U$ into $\mathbb{C}$. We say that $\mathcal{F}(U)$ is closed under division if, for each $f$ and $g$ in $\mathcal{F}(U)$, with $g \neq 0$ and $h=f / g$ a holomorphic function on $U$, we have $h \in \mathcal{F}(U)$.

The quotient notation $h=f / g$ means that $f(x)=h(x) \cdot g(x)$, for all $x \in U$.

Now we are able to enunciate two results for convolution equations defined on $\mathcal{H}_{\Theta b}(E)$ that we shall use, together with the techniques of Section 2, to obtain new existence and approximation results for convolution equations:

Theorem 3.10. 26. Theorem 4.2] If $\left(\mathcal{P}_{\Theta}\left({ }^{m} E\right)\right)_{m=0}^{\infty}$ is a $\pi_{1}-\pi_{2}$-holomorphy type, Exp $\Theta_{\Theta^{\prime}}\left(E^{\prime}\right)$ is closed under division and $L: \mathcal{H}_{\Theta b}(E) \longrightarrow \mathcal{H}_{\Theta b}(E)$ is a convolution operator, then the vector subspace of $\mathcal{H}_{\Theta b}(E)$ generated by the exponential polynomial solutions of the homogeneous equation $L=0$, is dense in the closed subspace of all solutions of the homogeneous equation, that is, the vector subspace of $\mathcal{H}_{\Theta b}(E)$ generated by

$$
\mathcal{L}=\left\{P \exp \varphi ; P \in \mathcal{P}_{\Theta}\left({ }^{m} E\right), m \in \mathbb{N}_{0}, \varphi \in E^{\prime}, L(P \exp \varphi)=0\right\}
$$

is dense in

$$
\operatorname{ker} L=\left\{f \in \mathcal{H}_{\Theta b}(E) ; L f=0\right\} .
$$

Theorem 3.11. [26, Theorem 4.4] If $\left(\mathcal{P}_{\Theta}\left({ }^{m} E\right)\right)_{m=0}^{\infty}$ is a $\pi_{1}-\pi_{2}$-holomorphy type, Exp $\operatorname{\Theta }_{\Theta^{\prime}}\left(E^{\prime}\right)$ is closed under division and $L: \mathcal{H}_{\Theta b}(E) \longrightarrow \mathcal{H}_{\Theta b}(E)$ is a non zero convolution operator, then $L$ is onto, that is, $L\left(\mathcal{H}_{\Theta b}(E)\right)=\mathcal{H}_{\Theta b}(E)$.

\section{Applications}

4.1. Lorentz nuclear and summing polynomials: the basics. For the sake of completeness we will recall the concepts of Lorentz summing polynomials introduced in 47] and Lorentz nuclear polynomials introduced in 28] and related results. We start introducing some notations.

We denote by $c_{0}(E)$ the Banach space (with the sup norm $\|\cdot\|_{\infty}$ ) composed by the sequences $\left(x_{j}\right)_{j=1}^{\infty}$ in the Banach space $E$ so that $\lim _{n \rightarrow \infty} x_{n}=0$, and $c_{00}(E)$ is the subspace of $c_{0}(E)$ formed by the sequences $\left(x_{j}\right)_{j=1}^{\infty}$ for which there is a $N_{0}$ such that $x_{n}=0$ for all $n \geq N_{0}$. When $E=\mathbb{K}:=\mathbb{R}$ or $\mathbb{C}$ we write $c_{0}$ and $c_{00}$ instead of $c_{0}(\mathbb{K})$ and $c_{00}(\mathbb{K})$, respectively. If $u=\left(u_{j}\right) \in c_{00}(E)$, the symbol $\operatorname{card}(u)$ denotes the cardinality of the set $\left\{j ; u_{j} \neq 0\right\}$.

As usual $\ell_{\infty}(E)$ represents the Banach space of bounded sequences in the Banach space $E$, with the sup norm and $\ell_{\infty}:=\ell_{\infty}(\mathbb{K})$. If $m \in \mathbb{N},\left(x_{j}\right)_{j=1}^{m}$ denotes $\left(x_{1}, \ldots, x_{m}, 0,0, \ldots\right)$, and when $\left(x_{j}\right)_{j=1}^{\infty}$ is a sequence of positive real numbers, we say that $\left(x_{j}\right)_{j=1}^{\infty}$ admits a non-increasing rearrangement if there is an injection $\pi: \mathbb{N} \rightarrow \mathbb{N}$ such that $x_{\pi(1)} \geq x_{\pi(2)} \geq \cdots$ and $x_{j}=x_{\pi(i)}$ for some $i$ whenever $x_{j} \neq 0$. If $p \geq 1$, then $p^{\prime}$ denotes the conjugate of $p$, i.e., $\frac{1}{p}+\frac{1}{p^{\prime}}=1$. 
Definition 4.1. 47] Let $E$ be a Banach space and $0<r, q<+\infty$.

(a) For $x=\left(x_{j}\right)_{j=1}^{\infty} \in \ell_{\infty}(E)$ we define

$$
a_{E, n}(x):=\inf \left\{\|x-u\|_{\infty} ; u \in c_{00}(E) \text { and } \operatorname{card}(u)<n\right\} .
$$

(b) The Lorentz sequence space $\ell_{(r, q)}(E)$ consists of all sequences $x=\left(x_{j}\right)_{j=1}^{\infty} \in \ell_{\infty}(E)$ such that

$$
\left(n^{\frac{1}{r}-\frac{1}{q}} a_{E, n}(x)\right)_{n=1}^{\infty} \in \ell_{q} .
$$

For $x \in \ell_{(r, q)}(E)$ we define the quasi-norm

$$
\|x\|_{(r, q)}=\left\|\left(n^{\frac{1}{r}-\frac{1}{q}} a_{E, n}(x)\right)_{n=1}^{\infty}\right\|_{q}
$$

(c) $\ell_{(r, q)}^{w}(E)$ is the space of all sequences $\left(x_{j}\right)_{j=1}^{\infty}$ in $E$ such that $\left\|\left(\varphi\left(x_{n}\right)\right)_{n=1}^{\infty}\right\|_{(r, q)}<\infty$ for every $\varphi \in E^{\prime}$. For $x \in \ell_{(r, q)}^{w}(E)$ we define the quasi-norm

$$
\left\|\left(x_{n}\right)_{n=1}^{\infty}\right\|_{w,(r, q)}:=\sup _{\|\varphi\| \leq 1}\left\|\left(\varphi\left(x_{n}\right)\right)_{n=1}^{\infty}\right\|_{(r, q)} .
$$

If endowed with the respective quasi-norms, $\ell_{(r, q)}(E)$ and $\ell_{(r, q)}^{w}(E)$ become complete spaces.

It is well known that $\ell_{(r, q)}(E) \subset c_{0}(E)$ and if $x=\left(x_{j}\right)_{j=1}^{\infty} \in c_{0}(E)$, then the sequence $\left(\left\|x_{j}\right\|\right)_{j=1}^{\infty}$ admits a non-increasing rearrangement.

Definition 4.2. [47, Definition 4.1] If $0<p, q, r, s<\infty$, an n-homogeneous polynomial $P \in \mathcal{P}\left({ }^{n} E ; F\right)$ is Lorentz $((s, p) ;(r, q))$-summing if $\left(P\left(x_{j}\right)\right)_{j=1}^{\infty} \in \ell_{(s, p)}(F)$ for each $\left(x_{j}\right)_{j=1}^{\infty} \in \ell_{(r, q)}^{w}(E)$.

The vector space composed by the Lorentz $((s, p) ;(r, q))$-summing $n$-homogeneous polynomials from $E$ to $F$ is denoted by $\mathcal{P}_{a s((s, p) ;(r, q))}\left({ }^{n} E ; F\right)$. When $n=1$ we write $\mathcal{L}_{a s((s, p) ;(r, q))}(E ; F)$.

When $s=p$, we write $\mathcal{L}_{a s(s ;(r, q))}$ instead of $\mathcal{L}_{a s((s, s) ;(r, q))}$; when $r=q$, we denote $\mathcal{L}_{a s((s, p) ; q)}$ instead of $\mathcal{L}_{a s((s, p) ;(q, q))}$.

Note that when $n=1, s=p$ and $r=q$ we have the usual concept of absolutely $(p ; q)$-summing operator. The space of absolutely $(p ; q)$-summing operators from $E$ to $F$ is represented by $\mathcal{L}_{a s(p ; q)}(E ; F)$. When $p=q$, we simply write $\mathcal{L}_{a s, p}$ instead of $\mathcal{L}_{a s(p ; q)}$. For the theory of absolutely summing linear operators we refer to [18].

Theorem 4.3. 47, Theorem 4.2] For $P \in \mathcal{P}\left({ }^{n} E ; F\right)$, the following conditions are equivalent:

(1) $P$ is Lorentz $((s, p) ;(r, q))$-summing.

(2) There is $C \geq 0$ such that

$$
\left\|\left(P\left(x_{j}\right)\right)_{j=1}^{m}\right\|_{(s, p)} \leq C\left\|\left(x_{j}\right)_{j=1}^{m}\right\|_{w,(r, q)}^{n}
$$

for all $m \in \mathbb{N}$ and $x_{1}, \ldots, x_{m} \in E$.

(3) There is $C \geq 0$ such that

$$
\left\|\left(P\left(x_{j}\right)\right)_{j=1}^{\infty}\right\|_{(s, p)} \leq C\left\|\left(x_{j}\right)_{j=1}^{\infty}\right\|_{w,(r, q)}^{n}
$$

for all $\left(x_{j}\right)_{j=1}^{\infty} \in \ell_{(r, q)}^{w}(E)$.

The infimum of the constants $C$ for which the above inequalities hold is a quasi-norm (denoted by $\left.\|.\|_{a s((s, p) ;(r, q))}\right)$ for $\mathcal{P}_{a s((s, p) ;(r, q))}\left({ }^{n} E ; F\right)$ and, under this quasi-norm, $\mathcal{P}_{a s((s, p) ;(r, q))}\left({ }^{n} E ; F\right)$ is complete.

Definition 4.4. 28, Definition 4.2] Let $E$ and $F$ be Banach spaces, $n \in \mathbb{N}$ and $r, q, s, p \in[1, \infty[$ such that $r \leq q, s^{\prime} \leq p^{\prime}$ and

$$
1 \leq \frac{1}{q}+\frac{n}{p^{\prime}}
$$


An n-homogeneous polynomial $P: E \rightarrow F$ is Lorentz $((r, q) ;(s, p))$-nuclear if

$$
P(x)=\sum_{j=1}^{\infty} \lambda_{j}\left(\varphi_{j}(x)\right)^{n} y_{j}
$$

with $\left(\lambda_{j}\right)_{j=1}^{\infty} \in \ell_{(r, q)},\left(\varphi_{j}\right)_{j=1}^{\infty} \in \ell_{\left(s^{\prime}, p^{\prime}\right)}^{w}\left(E^{\prime}\right)$ and $\left(y_{j}\right)_{j=1}^{\infty} \in \ell_{\infty}(F)$.

We denote by $\mathcal{P}_{N,((r, q) ;(s, p))}\left({ }^{n} E ; F\right)$ the subset of $\mathcal{P}\left({ }^{n} E ; F\right)$ composed by the $n$-homogeneous polynomials which are Lorentz $((r, q) ;(s, p))$-nuclear. We define

$$
\|P\|_{N,((r, q) ;(s, p))}=\inf \left\|\left(\lambda_{j}\right)_{j=1}^{\infty}\right\|_{(r, q)}\left\|\left(\varphi_{j}\right)_{j=1}^{\infty}\right\|_{w,\left(s^{\prime}, p^{\prime}\right)}^{n}\left\|\left(y_{j}\right)_{j=1}^{\infty}\right\|_{\infty},
$$

where the infimum is considered for all representations of $P \in \mathcal{P}_{N,((r, q) ;(s, p))}\left({ }^{n} E ; F\right)$ of the form (4.1).

Note that

$$
\|P\| \leq\|P\|_{N,((r, q) ;(s, p))} .
$$

From now on, unless stated otherwise, $r, q \in] 1, \infty\left[\right.$ and $s, p \in\left[1, \infty\left[\right.\right.$, with $r \leq q$ and $s^{\prime} \leq p^{\prime}$.

Proposition 4.5. [28, Propositions 4.3 and 4.4] The space $\left(\mathcal{P}_{N,((r, q) ;(s, p))}\left({ }^{n} E ; F\right),\|\cdot\|_{N,((r, q) ;(s, p))}\right)$ is a complete quasi-normed space. Besides, for $t_{n}$ given by

$$
\frac{1}{t_{n}}=\frac{1}{q}+\frac{n}{p^{\prime}}
$$

there is a $M \geq 0$ so that

$$
\|P+Q\|_{N,((r, q) ;(s, p))}^{t_{n}} \leq M\left(\|P\|_{N,((r, q) ;(s, p))}^{t_{n}}+\|Q\|_{N,((r, q) ;(s, p))}^{t_{n}}\right) .
$$

For this reason we call this quasi-norm a "quasi- $t_{n}$-norm".

Theorem 4.6. [28, Theorem 5.4] If $E^{\prime}$ has the bounded approximation property, then the linear mapping

$$
\Psi: \mathcal{P}_{N,((r, q) ;(s, p))}\left({ }^{n} E ; F\right)^{\prime} \rightarrow \mathcal{P}_{a s\left(\left(r^{\prime}, q^{\prime}\right) ;\left(s^{\prime}, p^{\prime}\right)\right)}\left({ }^{n} E^{\prime} ; F^{\prime}\right)
$$

given by $\Psi(T)=P_{T}$ is a topological isomorphism, where the map $P_{T}: E^{\prime} \rightarrow F^{\prime}$ is given by

$$
P_{T}(\varphi)(y)=T\left(\varphi^{n} y\right) .
$$

4.2. New hypercyclic, existence and approximation results. Now, suppose that $E^{\prime}$ has the bounded approximation property. The three steps below are common steps to obtain hypercyclic results (Theorems 3.5 and 3.6) and existence and approximation results (Theorems 3.10 and 3.11) for convolution operators:

(1) To obtain the spaces $\mathcal{P}_{\widetilde{N}},((r, q) ;(s, p))\left({ }^{n} E\right)$, for all $n \in \mathbb{N}$, according to Definition 2.2

(2) To prove that $\left(\mathcal{P}_{\widetilde{N},((r, q) ;(s, p))}\left({ }^{n} E\right)\right)_{n=0}^{\infty}$ is a holomorphy type.

(3) To prove that $\left(\mathcal{P}_{\widetilde{N},((r, q) ;(s, p))}\left({ }^{n} E\right)\right)_{n=0}^{\infty}$ is a $\pi_{1}-\pi_{2}$-holomorphy type.

A further step to obtain Theorems 3.10 and 3.11 is:

(4) To prove that $\operatorname{Exp}_{a s\left(\left(r^{\prime}, q^{\prime}\right) ;\left(s^{\prime}, p^{\prime}\right)\right)}\left(E^{\prime}\right)$ (see Definition 3.8(b)) is closed under division.

Step (1) is satisfied due to Proposition 4.5 and Theorem 4.6. In fact, Theorem 4.6 assures that the Borel transform is an isomorphism between $\mathcal{P}_{N,((r, q) ;(s, p))}\left({ }^{n} E ; F\right)^{\prime}$ and $\mathcal{P}_{a s}\left(\left(r^{\prime}, q^{\prime}\right) ;\left(s^{\prime}, p^{\prime}\right)\right)\left({ }^{n} E^{\prime} ; F^{\prime}\right)$. Thus, we can consider, for each $n \in \mathbb{N}$, the space $\mathcal{P}_{\widetilde{N}},((r, q) ;(s, p))\left({ }^{n} E\right)$, of all Lorentz $((r, q) ;(s, p))$-quasi-nuclear $n$-homogeneous polynomials from $E$ to $\mathbb{C}$, according to Definition 2.2 .

Now, let us prove Step (2), i.e., $\left(\mathcal{P}_{\widetilde{N},((r, q) ;(s, p))}\left({ }^{n} E\right)\right)_{n=0}^{\infty}$ is a holomorphy type. We only have to prove that $\left(\mathcal{P}_{\widetilde{N},((r, q) ;(s, p))}\left({ }^{n} E\right)\right)_{n=0}^{\infty}$ is stable for derivatives with constant $C_{n, k}=\frac{n !}{(n-k) !}$ (see Proposition 4.7 below) and the result follows from Corollary 2.9 
Proposition 4.7. If $P \in \mathcal{P}_{N,((r, q) ;(s, p))}\left({ }^{n} E ; F\right), k=1, \ldots, n$ and $x \in E$, then $\hat{d}^{k} P(x) \in \mathcal{P}_{N,((r, q) ;(s, p))}\left({ }^{k} E ; F\right)$ and

$$
\left\|\hat{d}^{k} P(x)\right\|_{N,((r, q) ;(s, p))} \leq \frac{n !}{(n-k) !}\|P\|_{N,((r, q) ;(s, p))}\|x\|^{n-k} .
$$

Proof. Let

$$
P(x)=\sum_{j=1}^{\infty} \lambda_{j}\left(\varphi_{j}(x)\right)^{n} y_{j},
$$

with $\left(\lambda_{j}\right)_{j=1}^{\infty} \in \ell_{(r, q)},\left(\varphi_{j}\right)_{j=1}^{\infty} \in \ell_{\left(s^{\prime}, p^{\prime}\right)}^{w}\left(E^{\prime}\right)$ and $\left(y_{j}\right)_{j=1}^{\infty} \in \ell_{\infty}(F)$. Then

$$
\hat{d}^{k} P(x)=\frac{n !}{(n-k) !} \sum_{j=1}^{\infty} \lambda_{j}\left(\varphi_{j}(x)\right)^{n-k} \varphi_{j}^{k} y_{j},
$$

for $k=1, \ldots, n$. Let $y=\frac{x}{\|x\|}$, and note that

$$
\left\|\left(\lambda_{j}\left(\varphi_{j}(y)\right)^{n-k}\right)_{j=1}^{\infty}\right\|_{(r, q)} \leq\left\|\left(\lambda_{j}\right)_{j=1}^{\infty}\right\|_{(r, q)} \sup _{j \in \mathbb{N}}\left|\varphi_{j}(y)\right|^{n-k} .
$$

Now we obtain

$$
\begin{aligned}
& \frac{n !}{(n-k) !}\|x\|^{n-k}\left\|\left(\lambda_{j}\left(\varphi_{j}(y)\right)^{n-k}\right)_{j=1}^{\infty}\right\|\left\|_{(r, q)}\right\|\left(\varphi_{j}\right)_{j=1}^{\infty}\left\|_{w,\left(s^{\prime}, p^{\prime}\right)}^{k}\right\|\left(y_{j}\right)_{j=1}^{\infty} \|_{\infty} \\
& \leq \frac{n !}{(n-k) !}\|x\|^{n-k}\left\|\left(\lambda_{j}\right)_{j=1}^{\infty}\right\| \sup _{(r, q)}\left|\varphi_{j}(y)\right|^{n-k}\left\|\left(\varphi_{j}\right)_{j=1}^{\infty}\right\|_{w,\left(s^{\prime}, p^{\prime}\right)}^{k}\left\|\left(y_{j}\right)_{j=1}^{\infty}\right\|_{\infty} \\
& \leq \frac{n !}{(n-k) !}\|x\|^{n-k}\left\|\left(\lambda_{j}\right)_{j=1}^{\infty}\right\|\left\|_{(r, q)}\right\|\left(\varphi_{j}\right)_{j=1}^{\infty}\left\|_{w,\left(s^{\prime}, p^{\prime}\right)}^{n-k}\right\|\left(\varphi_{j}\right)_{j=1}^{\infty}\left\|_{w,\left(s^{\prime}, p^{\prime}\right)}^{k}\right\|\left(y_{j}\right)_{j=1}^{\infty} \|_{\infty} \\
& =\frac{n !}{(n-k) !}\|x\|^{n-k}\left\|\left(\lambda_{j}\right)_{j=1}^{\infty}\right\|_{(r, q)}\left\|\left(\varphi_{j}\right)_{j=1}^{\infty}\right\|_{w,\left(s^{\prime}, p^{\prime}\right)}^{n}\left\|\left(y_{j}\right)_{j=1}^{\infty}\right\|_{\infty}<+\infty .
\end{aligned}
$$

Thus (4.2) is a valid Lorentz $((r, q) ;(s, p))$-nuclear representation of $\hat{d}^{k} P(x)$ and in view of the last inequalities we can write

$$
\left\|\hat{d}^{k} P(x)\right\|_{N,((r, q) ;(s, p))} \leq \frac{n !}{(n-k) !}\|x\|^{n-k}\left\|\left(\lambda_{j}\right)_{j=1}^{\infty}\right\|_{(r, q)}\left\|\left(\varphi_{j}\right)_{j=1}^{\infty}\right\|_{w,\left(s^{\prime}, p^{\prime}\right)}^{n}\left\|\left(y_{j}\right)_{j=1}^{\infty}\right\|_{\infty} .
$$

Hence

$$
\left\|\hat{d}^{k} P(x)\right\|_{N,((r, q) ;(s, p))} \leq \frac{n !}{(n-k) !}\|P\|_{N,((r, q) ;(s, p))}\|x\|^{n-k}
$$

as we wanted to show.

Now we are able to define the space of Lorentz $((r, q) ;(s, p))$-quasi-nuclear entire mappings of bounded type, according to Definition 3.1

Definition 4.8. An entire mapping $f: E \longrightarrow \mathbb{C}$ is said to be Lorentz $((r, q) ;(s, p))$-quasi-nuclear of bounded type if (1) $\hat{d}^{n} f(0) \in \mathcal{P}_{\tilde{N},((r, q) ;(s, p))}\left({ }^{n} E\right)$, for all $n \in \mathbb{N}_{0}$,

(2) $\lim _{n \rightarrow \infty}\left(\frac{1}{n !}\left\|\hat{d}^{n} f(0)\right\|_{\tilde{N},((r, q) ;(s, p))}\right)^{\frac{1}{n}}=0$.

The space of all entire mappings $f: E \longrightarrow \mathbb{C}$ that are Lorentz $((r, q) ;(s, p))$-quasi-nuclear of bounded type is denoted by $\mathcal{H}_{\tilde{N} b,((r, q) ;(s, p))}(E)$ and it is a Fréchet space with the topology generated by the family of seminorms:

$$
f \in \mathcal{H}_{\widetilde{N} b,((r, q) ;(s, p))}(E) \mapsto\|f\|_{\widetilde{N} b,((r, q) ;(s, p)), \rho}=\sum_{m=0}^{\infty} \frac{\rho^{m}}{m !}\left\|\hat{d}^{m} f(0)\right\|_{\widetilde{N},((r, q) ;(s, p))},
$$

for all $\rho>0$. 
Now we have to prove that $\left(\mathcal{P}_{\widetilde{N},((r, q) ;(s, p))}\left({ }^{n} E\right)\right)_{n=0}^{\infty}$ is a $\pi_{1}-\pi_{2}$-holomorphy type.

Proposition 4.9. $\left(\mathcal{P}_{\widetilde{N},((r, q) ;(s, p))}\left({ }^{n} E\right)\right)_{n=0}^{\infty}$ is a $\pi_{1}$-holomorphy type.

Proof. In [28, Example 4.5] it was proved that $\mathcal{P}_{f}\left({ }^{n} E\right)$ is contained in $\mathcal{P}_{N,((r, q) ;(s, p))}\left({ }^{n} E\right)$ and

$$
\left\|\phi^{n}\right\|_{N,((r, q) ;(s, p))}=\|\phi\|^{n}
$$

for all $\phi \in E^{\prime}$ and $n \in \mathbb{N}$. Besides, from [28, Lemma 4.10] we know that the space of finite type polynomials $\mathcal{P}_{f}\left({ }^{n} E\right)$ is dense in $\left(\mathcal{P}_{N,((r, q) ;(s, p))}\left({ }^{n} E\right),\|\cdot\|_{N,((r, q) ;(s, p))}\right)$. Thus, it follows from Proposition 2.5 that $\mathcal{P}_{f}\left({ }^{n} E\right)$ is dense in $\left(\mathcal{P}_{\widetilde{N},((r, q) ;(s, p))}\left({ }^{n} E\right),\|\cdot\|_{\widetilde{N},((r, q) ;(s, p))}\right)$ and

$$
\left\|\phi^{n}\right\|_{\widetilde{N},((r, q) ;(s, p))}=\|\phi\|^{n},
$$

for all $\phi \in E^{\prime}$ and $n \in \mathbb{N}$, since the constants $K$ and $C_{\Delta_{n}}$, in this case, may be taken equal to 1 .

Proposition 4.10. $\left(\mathcal{P}_{\widetilde{N},((r, q) ;(s, p))}\left({ }^{n} E\right)\right)_{n=0}^{\infty}$ is a $\pi_{2}$-holomorphy type.

Proof. Let $T \in\left[\mathcal{H}_{\tilde{N} b,((r, q) ;(s, p))}(E)\right]^{\prime}, n, k \in \mathbb{N}_{0}, k \leq n$ and $C, \rho>0$ be constants such that

$$
|T(f)| \leq C\|f\|_{\widetilde{N} b,((r, q) ;(s, p)), \rho} \text { for every } f \in \mathcal{H}_{\widetilde{N} b,((r, q) ;(s, p))}(E) .
$$

For $P \in \mathcal{P}_{\widetilde{N},((r, q) ;(s, p))}\left({ }^{n} E\right)$, we will show that the $(n-k)$-homogeneous polynomial

$$
\begin{aligned}
T\left(\widehat{A(\cdot)^{k}}\right): E & \longrightarrow \mathbb{C} \\
y & \mapsto T\left(A(\cdot)^{k} y^{n-k}\right)
\end{aligned}
$$

where $A: E^{n} \longrightarrow C$ is the unique continuous symmetric $n$-linear mapping such that $P=\hat{A}$, belongs to $\left.\mathcal{P}_{\widetilde{N},((r, q) ;(s, p))}{ }^{(n-k} E\right)$ and

$$
\left\|T\left(\widehat{A(\cdot)^{k}}\right)\right\|_{\widetilde{N},((r, q) ;(s, p))} \leq C \cdot \rho^{k}\|P\|_{\widetilde{N},((r, q) ;(s, p))} .
$$

First, suppose that $P \in \mathcal{P}_{N,((r, q) ;(s, p))}\left({ }^{n} E\right)$. Thus

$$
P=\sum_{j=1}^{\infty} \lambda_{j} \varphi_{j}^{n},
$$

with $\left(\lambda_{j}\right) \in \ell_{(r, q)}$ and $\left(\varphi_{j}\right) \in \ell_{\left(s^{\prime}, p^{\prime}\right)}^{w}\left(E^{\prime}\right)$, and for every $y \in E$ we have

$$
\begin{aligned}
T\left(\widehat{A(\cdot)^{k}}\right)(y) & =T\left(A(\cdot)^{k} y^{n-k}\right)=T\left(\sum_{j=1}^{\infty} \lambda_{j} \varphi_{j}^{k} \varphi_{j}(y)^{n-k}\right) \\
& =\sum_{j=1}^{\infty} \lambda_{j} T\left(\varphi_{j}^{k}\right) \varphi_{j}(y)^{n-k} .
\end{aligned}
$$

Now, to prove that $T\left(\widehat{A(\cdot)^{k}}\right) \in \mathcal{P}_{N,((r, q) ;(s, p))}\left({ }^{n-k} E\right)$, we only have to show that $\left(\lambda_{j} T\left(\varphi_{j}^{k}\right)\right) \in \ell_{(r, q)}$ since we already have $\left(\varphi_{j}\right) \in \ell_{\left(s^{\prime}, p^{\prime}\right)}^{w}\left(E^{\prime}\right)$. First, note that

$$
\begin{gathered}
\left\|\left(T\left(\varphi_{j}^{k}\right)\right)_{j=1}^{\infty}\right\|_{\infty}=\sup _{j}\left|T\left(\varphi_{j}^{k}\right)\right| \stackrel{\text { 4.5. }}{\leq} \sup _{j}\left\|\varphi_{j}^{k}\right\|_{\widetilde{N},((r, q) ;(s, p)), \rho} \\
\stackrel{(4.3)}{=} C \rho^{k} \sup _{j}\left\|\varphi_{j}^{k}\right\|_{\widetilde{N},((r, q) ;(s, p))} \stackrel{(4.4)}{=} C \rho^{k} \sup _{j}\left\|\varphi_{j}\right\|^{k} \\
=C \rho^{k}\left\|\left(\left\|\varphi_{j}\right\|\right)_{j=1}^{\infty}\right\|_{\infty}^{k} \leq C \rho^{k}\left\|\left(\varphi_{j}\right)_{j=1}^{\infty}\right\|_{w,\left(s^{\prime}, p^{\prime}\right)}^{k} .
\end{gathered}
$$


Hence

$$
\begin{aligned}
\left\|\left(\lambda_{j} T\left(\varphi_{j}^{k}\right)\right)_{j=1}^{\infty}\right\|_{(r, q)} & \leq\left\|\left(\lambda_{j}\right)_{j=1}^{\infty}\right\|_{(r, q)}\left\|\left(T\left(\varphi_{j}^{k}\right)\right)_{j=1}^{\infty}\right\|_{\infty} \\
& \leq C \rho^{k}\left\|\left(\lambda_{j}\right)_{j=1}^{\infty}\right\|_{(r, q)}\left\|\left(\varphi_{j}\right)_{j=1}^{\infty}\right\|_{w,\left(s^{\prime}, p^{\prime}\right)}^{k}<\infty
\end{aligned}
$$

and it follows that $T\left(\widehat{A(\cdot)^{k}}\right) \in \mathcal{P}_{N,((r, q) ;(s, p))}\left({ }^{n-k} E\right)$. Moreover

$$
\begin{aligned}
\left\|T\left(\widehat{A(\cdot)^{k}}\right)\right\|_{N,((r, q) ;(s, p))} & \leq\left\|\left(\lambda_{j} T\left(\varphi_{j}^{k}\right)\right)_{j=1}^{\infty}\right\|_{(r, q)}\left\|\left(\varphi_{j}\right)_{j=1}^{\infty}\right\|_{w,\left(s^{\prime}, p^{\prime}\right)}^{n-k} \\
& \leq\left[C \rho^{k}\left\|\left(\lambda_{j}\right)_{j=1}^{\infty}\right\|_{(r, q)}\left\|\left(\varphi_{j}\right)_{j=1}^{\infty}\right\|_{w,\left(s^{\prime}, p^{\prime}\right)}^{k}\right]\left\|\left(\varphi_{j}\right)_{j=1}^{\infty}\right\|_{w,\left(s^{\prime}, p^{\prime}\right)}^{n-k} \\
& =C \rho^{k}\left\|\left(\lambda_{j}\right)_{j=1}^{\infty}\right\|_{(r, q)}\left\|\left(\varphi_{j}\right)_{j=1}^{\infty}\right\|_{w,\left(s^{\prime}, p^{\prime}\right)}^{n} .
\end{aligned}
$$

and so

$$
\left\|T\left(\widehat{A(\cdot)^{k}}\right)\right\|_{\widetilde{N},((r, q) ;(s, p))} \stackrel{(\underline{2.1})}{\leq}\left\|T\left(\widehat{A(\cdot)^{k}}\right)\right\|_{N,((r, q) ;(s, p))} \leq C \rho^{k}\|P\|_{N,((r, q) ;(s, p))} .
$$

Now, if we define

$$
\begin{aligned}
\psi: \mathcal{P}_{N,((r, q) ;(s, p))}\left({ }^{n} E\right) & \rightarrow \mathcal{P}_{N,((r, q) ;(s, p))}\left({ }^{n-k} E\right) \\
P & \mapsto T\left(\widehat{A(\cdot)^{k}}\right)
\end{aligned}
$$

and proceeding in a similar way as in the proof of Theorem 2.8 , the result follows.

Now we can state the hypercyclicity results in this new framework:

Theorem 4.11. Let $E^{\prime}$ be separable. Then every convolution operator on $\mathcal{H}_{\widetilde{N} b,((r, q) ;(s, p))}(E)$ which is not a scalar multiple of the identity is hypercyclic.

Theorem 4.12. Let $E^{\prime}$ be separable and $T \in\left[\mathcal{H}_{\widetilde{N} b,((r, q) ;(s, p))}(E)\right]^{\prime}$ be a linear functional which is not a scalar multiple of $\delta_{0}$. Then $\bar{\Gamma}_{N,((r, q) ;(s, p))}(T)$ is a convolution operator that is not a scalar multiple of the identity, hence hypercyclic.

Remark 4.13. A recent result of Pinasco, Muro, Savransky [49] shows that nontrivial convolution operators on certain spaces of entire functions on a Banach space are strongly mixing in the gaussian sense, in particular frequently hypercyclic. We believe that, using the corresponding auxiliary results from [5], their result holds for $\mathcal{H}_{\Theta b}(E)$, when $\Theta$ is a $\pi_{1}$-holomorphy type. In this case, the reasoning used to prove the two theorems above actually proves that the convolution operators of the two theorems above can be proved to be strongly mixing in the gaussian sense.

Now, according to Definition 3.8(b) we introduce the Lorentz summing functions of exponential type:

Definition 4.14. An entire mapping $f: E \longrightarrow \mathbb{C}$ is said to be of Lorentz $((s, p) ;(r, q))$-summing exponential type if $\hat{d}^{n} f(0) \in \mathcal{P}_{\text {as }((s, p) ;(r, q))}\left({ }^{n} E\right)$, for all $n \in \mathbb{N}_{0}$, and there are $C \geq 0$ and $c>0$ such that

for all $n \in \mathbb{N}_{0}$.

$$
\left\|\hat{d}^{n} f(0)\right\|_{a s((s, p) ;(r, q))} \leq C c^{n}
$$

The vector space of all these mappings is denoted by $\operatorname{Exp}_{a s((s, p) ;(r, q))}(E)$.

This definition was motivated by the definition of mappings of exponential type (see [33]): 
Definition 4.15. An entire mapping $f: E \longrightarrow F$ is said to be of exponential type if one of the following equivalent conditions holds:

(i) There are $C \geq 0$ and $c>0$ such that $\|f(x)\| \leq C \exp (c\|x\|)$, for all $x \in E$.

(ii) There are $D \geq 0$ and $d>0$ such that $\left\|\hat{d}^{m} f(0)\right\| \leq C c^{m}$, for all $m \in \mathbb{N}$.

(iii) $\lim \sup _{m \rightarrow \infty}\left\|\hat{d}^{m} f(0)\right\|^{\frac{1}{m}}<+\infty$.

We denote by $\operatorname{Exp}(E ; F)$ the vector space of all entire mappings of exponential type from $E$ into $F$. When $F$ is the scalar field $\mathbb{C}$ we denote $\operatorname{Exp}(E)$ instead of $\operatorname{Exp}(E ; \mathbb{C})$.

The next two lemmata will be necessary for the proof of the division theorem (Theorem 4.18). The first is a division result due to Gupta (see [33, 34]) .

Lemma 4.16. If $f, g$ and $h$ are entire mappings on $E$ with values in $\mathbb{C}, f \neq 0, h(x)=f(x) g(x)$ for all $x \in E$, with $f$ and $h$ of exponential type on $E$, then $g$ is of exponential type on $E$.

Lemma 4.17. Let $r, q, s, p \in[1,+\infty[$ with $r \leq q$ and $F$ be a Banach space. If $f \in \operatorname{Exp}(F)$ and $g \in \operatorname{Exp}_{a s\left(\left(r^{\prime}, q^{\prime}\right) ;\left(s^{\prime}, p^{\prime}\right)\right)}(F)$ then $f g$ is in $\operatorname{Exp}_{a s\left(\left(r^{\prime}, q^{\prime}\right) ;\left(s^{\prime}, p^{\prime}\right)\right)}(F)$.

Proof. For each $k \in \mathbb{N}$, it follows from the uniqueness of the power series of a holomorphic function around a point of its domain that

$$
\hat{d}^{k}(f g)(0)(x)=\sum_{l=0}^{k} k ! \frac{1}{l !} \hat{d}^{l} f(0)(x) \frac{1}{(k-l) !} \hat{d}^{k-l} g(0)(x)
$$

for all $x \in F$. Since $f \in \operatorname{Exp}(F)$, there are $C \geq 0$ and $c>0$ such that

$$
\left\|\hat{d}^{n} f(0)\right\| \leq C c^{n},
$$

for every $n \in \mathbb{N}$. For $\left\|\left(x_{j}\right)_{j=1}^{\infty}\right\|_{w,\left(s^{\prime}, p^{\prime}\right)} \leq 1$, we have $\left\|x_{j}\right\| \leq 1$ for every $j \in \mathbb{N}$ and so

$$
\left|\hat{d}^{n} f(0)\left(x_{j}\right)\right| \leq C c^{n},
$$

for every $n \in \mathbb{N}$. Thus

$$
\left|\hat{d}^{k}(f g)(0)\left(x_{j}\right)\right| \leq C \sum_{l=0}^{k} \frac{k !}{l !(k-l) !} c^{l}\left|\hat{d}^{k-l} g(0)\left(x_{j}\right)\right|,
$$

for all $j \in \mathbb{N}$. Let $\pi: \mathbb{N} \longrightarrow \mathbb{N}$ be an injection. Since $r^{\prime} \geq q^{\prime}$ we have (using Lemma 3.1 of [4])

$$
\begin{aligned}
{\left[\sum_{j=1}^{\infty}\left(j^{\frac{1}{r^{\prime}}-\frac{1}{q^{\prime}}}\left|\hat{d}^{k}(f g)(0)\left(x_{\pi(j)}\right)\right|\right)^{q^{\prime}}\right]^{\frac{1}{q^{\prime}}} } & \leq C \sum_{l=0}^{k} \frac{k !}{l !(k-l) !} c^{l}\left[\sum_{j=1}^{\infty}\left(j^{\frac{1}{r^{\prime}}-\frac{1}{q^{\prime}}}\left|\hat{d}^{k-l} g(0)\left(x_{\pi(j)}\right)\right|\right)^{q^{\prime}}\right]^{\frac{1}{q^{\prime}}} \\
& \leq C \sum_{l=0}^{k} \frac{k !}{l !(k-l) !} c^{l}\left\|\left(\hat{d}^{k-l} g(0)\left(x_{j}\right)\right)_{j=1}^{\infty}\right\|_{\left(r^{\prime}, q^{\prime}\right)} .
\end{aligned}
$$

By Theorem 4.3 we have

$$
\left\|\left(\hat{d}^{k-l} g(0)\left(x_{j}\right)\right)_{j=1}^{\infty}\right\|_{\left(r^{\prime}, q^{\prime}\right)} \leq\left\|\hat{d}^{k-l} g(0)\right\|_{a s\left(\left(r^{\prime}, q^{\prime}\right),\left(s^{\prime}, p^{\prime}\right)\right)}\left\|\left(x_{j}\right)_{j=1}^{\infty}\right\|_{w,\left(s^{\prime}, p^{\prime}\right)}^{k-l} \leq\left\|\hat{d}^{k-l} g(0)\right\|_{a s\left(\left(r^{\prime}, q^{\prime}\right),\left(s^{\prime}, p^{\prime}\right)\right)},
$$

for all $l=1, \ldots, k$. Thus

$$
\left[\sum_{j=1}^{\infty}\left(j^{\frac{1}{r^{\prime}}-\frac{1}{q^{\prime}}}\left|\hat{d}^{k}(f g)(0)\left(x_{\pi(j)}\right)\right|\right)^{q^{\prime}}\right]^{\frac{1}{q^{\prime}}} \leq C \sum_{l=0}^{k} \frac{k !}{l !(k-l) !} c^{l}\left\|\hat{d}^{k-l} g(0)\right\|_{a s\left(\left(r^{\prime}, q^{\prime}\right),\left(s^{\prime}, p^{\prime}\right)\right)}
$$

and we conclude that $\left(\left|\hat{d}^{k}(f g)(0)\left(x_{\pi(j)}\right)\right|\right)_{j=1}^{\infty} \in \ell_{\left(r^{\prime}, q^{\prime}\right)}$ and thus $\left(\hat{d}^{k}(f g)(0)\left(x_{\pi(j)}\right)\right)_{j=1}^{\infty} \in c_{0}$. 
Since $\pi$ is arbitrary we have

$$
\left[\sum_{j=1}^{\infty}\left(j^{\frac{1}{r^{\prime}}-\frac{1}{q^{\prime}}}\left|\hat{d}^{k}(f g)(0)\left(x_{\sigma(j)}\right)\right|\right)^{q^{\prime}}\right]^{\frac{1}{q^{\prime}}}=\left\|\left(\hat{d}^{k}(f g)(0)\left(x_{j}\right)\right)_{j=1}^{\infty}\right\|_{\left(r^{\prime}, q^{\prime}\right)}
$$

for some injection $\sigma: \mathbb{N} \longrightarrow \mathbb{N}$. Hence

$$
\left\|\left(\hat{d}^{k}(f g)(0)\left(x_{j}\right)\right)_{j=1}^{\infty}\right\|_{\left(r^{\prime}, q^{\prime}\right)} \leq C \sum_{l=0}^{k} \frac{k !}{l !(k-l) !} c^{l}\left\|\hat{d}^{k-l} g(0)\right\|_{a s\left(\left(r^{\prime}, q^{\prime}\right),\left(s^{\prime}, p^{\prime}\right)\right)} .
$$

Now, let $0 \neq\left(x_{j}\right)_{j=1}^{\infty} \in \ell_{\left(s^{\prime}, p^{\prime}\right)}^{w}(F)$. Defining

$$
y_{j}=\frac{x_{j}}{\left\|\left(x_{j}\right)_{j=1}^{\infty}\right\|_{w,\left(s^{\prime}, p^{\prime}\right)}},
$$

for all $j \in \mathbb{N}$, we have

$$
\left\|\left(y_{j}\right)_{j=1}^{\infty}\right\|_{w,\left(s^{\prime}, p^{\prime}\right)}=1
$$

and using the previous estimates we obtain

$$
\left\|\left(\hat{d}^{k}(f g)(0)\left(x_{j}\right)\right)_{j=1}^{\infty}\right\|_{\left(r^{\prime}, q^{\prime}\right)} \leq\left(C \sum_{l=0}^{k} \frac{k !}{l !(k-l) !} c^{l}\left\|\hat{d}^{k-l} g(0)\right\|_{a s\left(\left(r^{\prime}, q^{\prime}\right),\left(s^{\prime}, p^{\prime}\right)\right)}\right)\left\|\left(x_{j}\right)_{j=1}^{\infty}\right\|_{w,\left(s^{\prime}, p^{\prime}\right)}^{k} .
$$

Using again Theorem 4.3 it follows that $\hat{d}^{k} g(0) \in \mathcal{P}_{a s\left(\left(r^{\prime}, q^{\prime}\right) ;\left(s^{\prime}, p^{\prime}\right)\right)}\left({ }^{k} F\right)$ and

$$
\left\|\hat{d}^{k}(f g)(0)\right\|_{a s\left(\left(r^{\prime}, q^{\prime}\right),\left(s^{\prime}, p^{\prime}\right)\right)} \leq C \sum_{l=0}^{k} \frac{k !}{l !(k-l) !} c^{l}\left\|\hat{d}^{k-l} g(0)\right\|_{a s\left(\left(r^{\prime}, q^{\prime}\right),\left(s^{\prime}, p^{\prime}\right)\right)} .
$$

Since $g \in \operatorname{Exp}_{a s\left(\left(r^{\prime}, q^{\prime}\right) ;\left(s^{\prime}, p^{\prime}\right)\right)}(F)$ there are $D \geq 0$ and $d>0$ such that

$$
\left\|\hat{d}^{n} g(0)\right\|_{a s\left(\left(r^{\prime}, q^{\prime}\right),\left(s^{\prime}, p^{\prime}\right)\right)} \leq D d^{n},
$$

for all $n \in \mathbb{N}$. Hence

$$
\left\|\hat{d}^{k}(f g)(0)\right\|_{a s\left(\left(r^{\prime}, q^{\prime}\right),\left(s^{\prime}, p^{\prime}\right)\right)} \leq C D \sum_{l=0}^{k} \frac{k !}{l !(k-l) !} c^{l} d^{k-l}=C D(c+d)^{k}
$$

and $f g \in \operatorname{Exp}_{a s\left(\left(r^{\prime}, q^{\prime}\right) ;\left(s^{\prime}, p^{\prime}\right)\right)}(F)$.

Theorem 4.18 (Division Theorem). Let $r, q, s, p \in[1,+\infty[$ with $r \leq q$ and $F$ be a Banach space. Then $\operatorname{Exp}_{\text {as }\left(\left(r^{\prime}, q^{\prime}\right) ;\left(s^{\prime}, p^{\prime}\right)\right)}(F)$ is closed under division, that is, if $f, g$ and $h$ are entire mappings on $F$ with values in $\mathbb{C}, f \neq 0$, and $h=f g$ with $f$ and $h$ in $\operatorname{Exp}_{a s\left(\left(r^{\prime}, q^{\prime}\right) ;\left(s^{\prime}, p^{\prime}\right)\right)}(F)$, then $g$ is also in $\operatorname{Exp}_{a s\left(\left(r^{\prime}, q^{\prime}\right) ;\left(s^{\prime}, p^{\prime}\right)\right)}(F)$.

Proof. For each $k \in \mathbb{N}$ we have

$$
\hat{d}^{k} h(0)(x)=f(0) \hat{d}^{k} g(0)(x)+\sum_{l=1}^{k} \frac{k !}{l !(k-l) !} \hat{d}^{l} f(0)(x) \hat{d}^{k-l} g(0)(x)
$$

and so

$$
f(0) \hat{d}^{k} g(0)(x)=\hat{d}^{k} h(0)(x)-\sum_{l=1}^{k} \frac{k !}{l !(k-l) !} \hat{d}^{l} f(0)(x) \hat{d}^{k-l} g(0)(x)
$$

for all $x \in F$. Let us suppose $f(0) \neq 0$. For $\left\|\left(x_{j}\right)_{j=1}^{\infty}\right\|_{w,\left(s^{\prime}, p^{\prime}\right)} \leq 1$, we have $\left\|x_{j}\right\| \leq 1$ for every $j \in \mathbb{N}$. Since $\|\cdot\| \leq\|\cdot\|_{a s\left(\left(r^{\prime}, q^{\prime}\right) ;\left(s^{\prime}, p^{\prime}\right)\right)}$, it follows from Definition 4.15)(ii) that $g$ is of exponential type. So there are $C \geq 0$ and $c>0$ such that

$$
\left\|\hat{d}^{k} g(0)\right\| \leq C c^{k} .
$$


Therefore

$$
\left|\hat{d}^{k} g(0)\left(x_{j}\right)\right| \leq \frac{1}{|f(0)|}\left|\hat{d}^{k} h(0)\left(x_{j}\right)\right|+\frac{C}{|f(0)|} \sum_{l=1}^{k} \frac{k !}{l !(k-l) !} c^{k-l}\left|\hat{d}^{l} f(0)\left(x_{j}\right)\right|,
$$

for every $j \in \mathbb{N}$. Let $\pi: \mathbb{N} \longrightarrow \mathbb{N}$ be an injection. Since $r^{\prime} \geq q^{\prime}$ we have (using Lemma 3.1 of [47])

$$
\begin{aligned}
& {\left[\sum_{j=1}^{\infty}\left(j^{\frac{1}{r^{\prime}}-\frac{1}{q^{\prime}}}\left|\hat{d}^{k} g(0)\left(x_{\pi(j)}\right)\right|\right)^{q^{\prime}}\right]^{\frac{1}{q^{\prime}}}} \\
& \leq \frac{1}{|f(0)|}\left[\sum_{j=1}^{\infty}\left(j^{\frac{1}{r^{\prime}}-\frac{1}{q^{\prime}}}\left|\hat{d}^{k} h(0)\left(x_{\pi(j)}\right)\right|\right)^{q^{\prime}}\right]^{\frac{1}{q^{\prime}}} \\
& +\frac{C}{|f(0)|} \sum_{l=1}^{k} \frac{k !}{l !(k-l) !} c^{k-l}\left[\sum_{j=1}^{\infty}\left(j^{\frac{1}{r^{\prime}}-\frac{1}{q^{\prime}}}\left|\hat{d}^{l} f(0)\left(x_{\pi(j)}\right)\right|\right)^{q^{\prime}}\right]^{\frac{1}{q^{\prime}}} \\
& \leq \frac{1}{|f(0)|}\left\|\left(\hat{d}^{k} h(0)\left(x_{j}\right)\right)_{j=1}^{\infty}\right\|_{\left(r^{\prime}, q^{\prime}\right)}+\frac{C}{|f(0)|} \sum_{l=1}^{k} \frac{k !}{l !(k-l) !} c^{k-l}\left\|\left(\hat{d}^{l} f(0)\left(x_{j}\right)\right)_{j=1}^{\infty}\right\|_{\left(r^{\prime}, q^{\prime}\right)} .
\end{aligned}
$$

By Theorem 4.3 we have

$$
\begin{aligned}
& \left\|\left(\hat{d}^{k} h(0)\left(x_{j}\right)\right)_{j=1}^{\infty}\right\|_{\left(r^{\prime}, q^{\prime}\right)} \leq\left\|\hat{d}^{k} h(0)\right\|_{a s\left(\left(r^{\prime}, q^{\prime}\right),\left(s^{\prime}, p^{\prime}\right)\right)}\left\|\left(x_{j}\right)_{j=1}^{\infty}\right\|_{w,\left(s^{\prime}, p^{\prime}\right)}^{k} \leq\left\|\hat{d}^{k} h(0)\right\|_{a s\left(\left(r^{\prime}, q^{\prime}\right),\left(s^{\prime}, p^{\prime}\right)\right)}, \\
& \left\|\left(\hat{d}^{l} f(0)\left(x_{j}\right)\right)_{j=1}^{\infty}\right\|_{\left(r^{\prime}, q^{\prime}\right)} \leq\left\|\hat{d}^{l} f(0)\right\|_{a s\left(\left(r^{\prime}, q^{\prime}\right),\left(s^{\prime}, p^{\prime}\right)\right)}\left\|\left(x_{j}\right)_{j=1}^{\infty}\right\|_{w,\left(s^{\prime}, p^{\prime}\right)}^{l} \leq\left\|\hat{d}^{l} f(0)\right\|_{a s\left(\left(r^{\prime}, q^{\prime}\right),\left(s^{\prime}, p^{\prime}\right)\right)},
\end{aligned}
$$

for all $l=1, \ldots, k$. Thus

$$
\begin{aligned}
& {\left[\sum _ { j = 1 } ^ { \infty } \left(j^{\left.\left.\frac{1}{r^{\prime}}-\frac{1}{q^{\prime}}\left|\hat{d}^{k} g(0)\left(x_{\pi(j)}\right)\right|\right)^{q^{\prime}}\right]^{\frac{1}{q^{\prime}}}}\right.\right.} \\
& \leq \frac{1}{|f(0)|}\left\|\hat{d}^{k} h(0)\right\|_{a s\left(\left(r^{\prime}, q^{\prime}\right),\left(s^{\prime}, p^{\prime}\right)\right)}+\frac{C}{|f(0)|} \sum_{l=1}^{k} \frac{k !}{l !(k-l) !} c^{k-l}\left\|\hat{d}^{l} f(0)\right\|_{a s\left(\left(r^{\prime}, q^{\prime}\right),\left(s^{\prime}, p^{\prime}\right)\right)} .
\end{aligned}
$$

We conclude that $\left(\left|\hat{d}^{k} g(0)\left(x_{\pi(j)}\right)\right|\right)_{j=1}^{\infty} \in \ell_{\left(r^{\prime}, q^{\prime}\right)}$ and thus $\left(\hat{d}^{k} g(0)\left(x_{\pi(j)}\right)\right)_{j=1}^{\infty} \in c_{0}$. Since $\pi$ is arbitrary we have

$$
\left[\sum_{j=1}^{\infty}\left(j^{\frac{1}{r^{\prime}}-\frac{1}{q^{\prime}}}\left|\hat{d}^{k} g(0)\left(x_{\sigma(j)}\right)\right|\right)^{q^{\prime}}\right]^{\frac{1}{q^{\prime}}}=\left\|\left(\hat{d}^{k} g(0)\left(x_{j}\right)\right)_{j=1}^{\infty}\right\|_{\left(r^{\prime}, q^{\prime}\right)}
$$

for some injection $\sigma: \mathbb{N} \longrightarrow \mathbb{N}$.

Now, let $0 \neq\left(x_{j}\right)_{j=1}^{\infty} \in \ell_{\left(s^{\prime}, p^{\prime}\right)}^{w}(F)$. Defining

$$
y_{j}=\frac{x_{j}}{\left\|\left(x_{j}\right)_{j=1}^{\infty}\right\|_{w,\left(s^{\prime}, p^{\prime}\right)}},
$$

for all $j \in \mathbb{N}$, we have $\left\|\left(y_{j}\right)_{j=1}^{\infty}\right\|_{w,\left(s^{\prime}, p^{\prime}\right)}=1$ and using the previous estimates we obtain

$$
\begin{aligned}
& \left\|\left(\hat{d}^{k} g(0)\left(x_{j}\right)\right)_{j=1}^{\infty}\right\|_{\left(r^{\prime}, q^{\prime}\right)} \\
& \leq\left(\frac{1}{|f(0)|}\left\|\hat{d}^{k} h(0)\right\|_{a s\left(\left(r^{\prime}, q^{\prime}\right),\left(s^{\prime}, p^{\prime}\right)\right)}+\frac{C}{|f(0)|} \sum_{l=1}^{k} \frac{k !}{l !(k-l) !} c^{k-l}\left\|\hat{d}^{l} f(0)\right\|_{a s\left(\left(r^{\prime}, q^{\prime}\right),\left(s^{\prime}, p^{\prime}\right)\right)}\right)\left\|\left(x_{j}\right)_{j=1}^{\infty}\right\|_{w,\left(s^{\prime}, p^{\prime}\right)}^{k} .
\end{aligned}
$$


Using again Theorem 4.3, it follows that $\hat{d}^{k} g(0) \in \mathcal{P}_{a s\left(\left(r^{\prime}, q^{\prime}\right) ;\left(s^{\prime}, p^{\prime}\right)\right)}\left({ }^{k} F\right)$. Since $f, h \in \operatorname{Exp}_{a s\left(\left(r^{\prime}, q^{\prime}\right) ;\left(s^{\prime}, p^{\prime}\right)\right)}(F)$, there are $A, B \geq 0$ and $a, b>0$ such that

$$
\left\|\hat{d}^{k} h(0)\right\|_{a s\left(\left(r^{\prime}, q^{\prime}\right),\left(s^{\prime}, p^{\prime}\right)\right)} \leq A a^{k}
$$

and

$$
\left\|\hat{d}^{l} f(0)\right\|_{a s\left(\left(r^{\prime}, q^{\prime}\right),\left(s^{\prime}, p^{\prime}\right)\right)} \leq B b^{l}
$$

for $l=1, \ldots, k$. Hence

$$
\begin{aligned}
\left\|\left(\hat{d}^{k} g(0)\left(x_{j}\right)\right)_{j=1}^{\infty}\right\|_{\left(r^{\prime}, q^{\prime}\right)} & \leq\left(\frac{A a^{k}}{|f(0)|}+\frac{C B}{|f(0)|} \sum_{l=1}^{k} k ! \frac{1}{l !} \frac{1}{(k-l) !} c^{k-l} b^{l}\right)\left\|\left(x_{j}\right)_{j=1}^{\infty}\right\|_{w,\left(s^{\prime}, p^{\prime}\right)}^{k} \\
& \leq\left(\frac{A}{|f(0)|}+\frac{C B}{|f(0)|}\right)(a+b+c)^{k}\left\|\left(x_{j}\right)_{j=1}^{\infty}\right\|_{w,\left(s^{\prime}, p^{\prime}\right)}^{k},
\end{aligned}
$$

for all $\left(x_{j}\right)_{j=1}^{\infty} \in \ell_{\left(s^{\prime}, p^{\prime}\right)}^{w}(F)$ and by the definition of $\|\cdot\|_{a s\left(\left(r^{\prime}, q^{\prime}\right),\left(s^{\prime}, p^{\prime}\right)\right)}$ (see Theorem 4.3) we have

$$
\left\|\hat{d}^{k} g(0)\right\|_{a s\left(\left(r^{\prime}, q^{\prime}\right),\left(s^{\prime}, p^{\prime}\right)\right)} \leq D d^{k},
$$

with $D=\frac{A+B C}{|f(0)|} \geq 0$ and $d=a+b+c \geq 0$.

Now, suppose that $f(0)=0$ and define

$$
f_{0}(x)=f(x)+\psi(x)
$$

and

$$
h_{0}(x)=h(x)+\psi(x) g(x)
$$

for all $x \in F$, where $\psi \in \operatorname{Exp}_{a s\left(\left(r^{\prime}, q^{\prime}\right) ;\left(s^{\prime}, p^{\prime}\right)\right)}(F), \psi(0) \neq 0$ and $\psi$ is non constant (for example, let $\psi(x)=1+P(x)$, with $P \in \mathcal{P}_{a s}((s, p) ;(r, q))\left({ }^{n} F\right)$ for some $\left.n \neq 0\right)$. Thus $h_{0}=f_{0} g, f_{0}(0) \neq 0$, $f_{0} \in \operatorname{Exp}_{a s\left(\left(r^{\prime}, q^{\prime}\right) ;\left(s^{\prime}, p^{\prime}\right)\right)}(F)$ and by Lemma 4.17 $h_{0} \in \operatorname{Exp}_{a s\left(\left(r^{\prime}, q^{\prime}\right) ;\left(s^{\prime}, p^{\prime}\right)\right)}(F)$. Applying the result we have just proved, it follows that $g \in \operatorname{Exp}_{a s\left(\left(r^{\prime}, q^{\prime}\right) ;\left(s^{\prime}, p^{\prime}\right)\right)}(F)$.

For $F=E^{\prime}$, we have that $\operatorname{Exp}_{a s\left(\left(r^{\prime}, q^{\prime}\right) ;\left(s^{\prime}, p^{\prime}\right)\right)}\left(E^{\prime}\right)$ is closed under division, and so it follows from Theorems 3.10 and 3.11 that we have existence and approximation results for convolution equations defined on $\mathcal{H}_{\widetilde{N} b,((r, q) ;(s, p))}(E)$ as enunciated below.

Theorem 4.19. The vector subspace of $\mathcal{H}_{\widetilde{N} b,((r, q) ;(s, p))}(E)$, generated by the exponential polynomial solutions of the homogeneous equation $L=0$, is dense in the closed subspace of all solutions of the homogeneous equation, that is, the vector subspace of $\mathcal{H}_{\widetilde{N} b,((r, q) ;(s, p))}(E)$ generated by

$$
\mathcal{L}=\left\{P \exp \varphi ; P \in \mathcal{P}_{\widetilde{N}},((r, q) ;(s, p))\left({ }^{m} E\right), m \in \mathbb{N}_{0}, \varphi \in E^{\prime}, L(P \exp \varphi)=0\right\}
$$

is dense in

$$
\operatorname{ker} L=\left\{f \in \mathcal{H}_{\widetilde{N} b,((r, q) ;(s, p))}(E) ; L f=0\right\} .
$$

Theorem 4.20. If $L$ is a non zero convolution operator, then

$$
L\left(\mathcal{H}_{\widetilde{N} b,((r, q) ;(s, p))}(E)\right)=\mathcal{H}_{\widetilde{N} b,((r, q) ;(s, p))}(E) .
$$

Acknowledgements. The authors thank Mário C. Matos for introducing them to this subject and for his permanent encouragement. The authors also want to thank G. Botelho for his useful suggestions. 


\section{REFERENCES}

[1] R. Aron and D. Markose, On universal functions, in: Satellite Conference on Infinite Dimensional Function Theory, J. Korean Math. Soc. 41 (2004), 65-76.

[2] F. Bayart, É. Matheron, Dynamics of linear operators, Cambridge Tracts in Mathematics, 179. Cambridge University Press, Cambridge, 2009.

[3] F. Bayart, É. Matheron, Mixing operators and small subsets of the circle, J. Reine Angew. Math., to appear.

[4] L. Bernal-Gonzalez, D. Pellegrino, J.B. Seoane-Sepulveda, Linear subsets of nonlinear sets in topological vector spaces, Bull. Amer. Math. Soc. (N.S.) 51 (2014), no. 1, 71-130.

[5] F. J. Bertoloto, G. Botelho, V. V. Fávaro, A. M. Jatobá, Hypercyclicity of convolution operators on spaces of entire functions. Ann. Inst. Fourier (Grenoble) 63 (2013), 1263-1283.

[6] J. Bès, Ö. Martin, A. Peris and S. Shkarin Disjoint mixing operators, J. Funct. Anal. 263 (2012), 1283-1322.

[7] G. D. Birkhoff, Démonstration d'un théorème élémentaire sur les fonctions entières, C. R. Acad. Sci. Paris 189 (1929), 473-475.

[8] C. Boyd, Duality and reflexivity of spaces of approximable polynomials on locally convex spaces, Monatsh. Math. 130 (2000), 177-188.

[9] C. Boyd and A. Brown, Duality in spaces of polynomials pf degree at most n, J. Math. Anal. Appl. 429 (2015), 1271-1290.

[10] C. Boyd S. Dineen and P. Rueda, Locally Asplund space of holomorphic functions, Michigan Math. J. 50 (2002), 493-506.

[11] D. Carando and V. Dimant, Duality in spaces of nuclear and integral polynomials, J. Math. Anal. Appl. 241 (2000), 107-121.

[12] D. Carando, V. Dimant and S. Muro, Hypercyclic convolution operators on Fréchet spaces of analytic functions, J. Math. Anal. Appl. 336 (2007), 1324-1340.

[13] K. C. Chan and J. H. Shapiro, The cyclic behaviour of translation operators on Hilbert spaces of entire funcitons, Indiana Univ. J. 40 (1991), 1421-1449.

[14] J. F. Colombeau and M. C. Matos, Convolution equations in spaces of infinite dimensional entire functions, Indag. Math. 42 (1980), 375-389.

[15] J.F Colombeau and B. Perrot, Convolution equations in spaces of infinite dimensional entire functions of exponencial and related types, Trans. Amer. Math. Soc. 258 (1980), 191-198.

[16] J.F Colombeau, R. Gay and B. Perrot, Division by holomorphic functions and convolution equations in infinite dimension, Trans. Amer. Math. Soc. 264 (1981), 381-391.

[17] A. Defant and K. Floret, Tensor Norms and Operator Ideals, North-Holland Math. Studies 176, 1993.

[18] J. Diestel, H. Jarchow and A. Tonge, Absolutely Summing Operators, Cambridge Studies in Advanced Mathematics 43, 1995.

[19] S. Dineen, Complex Analysis on Infinite Dimensional Spaces, Springer-Verlag, London, 1999.

[20] S. Dineen, Holomorphy types on a Banach space, Studia Math. 39 (1971), 241-288.

[21] J. Dieudonné and L. Schwartz, La dualité dans les espaces $(\mathcal{F})$ et $(\mathcal{L F})$, Ann. Inst. Fourier (Grenoble) I (1949), 61-101.

[22] T.A.W. Dwyer III, Convolution equations for vector-valued entire functions of nuclear bounded type, Trans. Amer. Math. Soc. 217 (1976), 105- 119.

[23] T.A.W. Dwyer III, Partial differential equations in Fischer-Fock spaces for the Hilbert-Schmidt holomorphy type, Bull. Amer. Math. Soc. 77 (1971), 725- 730.

[24] V. V. Fávaro, The Fourier-Borel transform between spaces of entire functions of a given type and order, Portugal. Math. 65 (2008), 285-309.

[25] V. V. Fávaro, Convolution equations on spaces of quasi-nuclear functions of a given type and order, Bull. Belg. Math. Soc. Simon Stevin 17 (2010), 535-569.

[26] V. V. Fávaro, A. M. Jatobá, Holomorphy types and spaces of entire functions of bounded type on Banach spaces, Czech. Math. Journal 59 (2009), 909-927.

[27] V. V. Fávaro, A. M. Jatobá, Holomorphy types and the Fourier-Borel transform between spaces of entire functions of a given type and order defined on Banach spaces, Math. Scand. 110 (2012), 111-139.

[28] V. V. Fávaro, M. C. Matos, D. Pellegrino, On Lorentz nuclear homogeneous polynomials between Banach spaces, Portugal. Math. 67 (2010), 413-435.

[29] V. V. Fávaro, J. Mujica, Hypercyclic convolution operators on spaces of entire functions, to appear in J. Operator Theory.

[30] R. M. Gethner and J. H. Shapiro, Universal vectors for operators on spaces of holomorphic functions, Proc. Amer. Math. Soc. 100 (1987), 281-288.

[31] G. Godefroy and J. H. Shapiro, Operators with dense, invariant, cyclic vector manifolds, J. Funct. Anal. 98 (1991), 229-269.

[32] K. G. Grosse-Erdmann, Universal families and hypercyclic operators, Bull. Amer. Math. Soc. 36 (1999), 345-381. 
[33] C. P. Gupta, Convolution Operators and Holomorphic Mappings on a Banach Space, Seminaire d'Analyse Moderne, 2, Université de Sherbrooke, Sherbrooke, 1969.

[34] C. P. Gupta, On the Malgrange Theorem for nuclearly entire functions of bounded type on a Banach space, Indag. Math. 32 (1970), 356-358.

[35] L. Hörmander, On the division of distributions by polynomials, Ark. Math. 3 (1958), 555-568.

[36] C. Kitai, Invariant closed sets for linear operators, Dissertation, University of Toronto, 1982.

[37] S. Lojasiewicz, Sur le problème de la division, Studia Math. 18 (1959), 87-136.

[38] G. R. MacLane, Sequences of derivatives and normal families, J. Anal. Math. 2 (1952), 72-87.

[39] B. Malgrange, Existence et approximation des solutions des équations aux derivées partielles et des équations des convolutions, Ann. Inst. Fourier (Grenoble) 6 (1955-56), 271-355.

[40] A. Martineau, Équations différentielles d'ordre infini, Bull. Soc. Math. France 95 (1967), 109-154.

[41] M. C. Matos, Sur le théorème d'approximatin et d'existence de Malgrange-Gupta, C. R. Acad. Sci. Paris, 271 (1970), 1258-1259.

[42] M. C. Matos, On Malgrange Theorem for nuclear holomorphic functions in open balls of a Banach space, Math. Z. 171 (1980), 113-123.

[43] M. C. Matos, Correction to "On Malgrange Theorem for nuclear holomorphic functions in open balls of a Banach space", Math. Z. (1980), 289-290.

[44] M. C. Matos, On convolution operators in spaces of entire functions of a given type and order, in: Complex Analysis, Functional Analysis and Approximation Theory (J. Mujica, ed.), pp. 129-171. North-Holland Math. Studies 125, North-Holland, Amsterdam, 1986.

[45] M. C. Matos, Absolutely summing mappings, nuclear mappings and convolution equations, IMECC-UNICAMP, 2007. Web: http://www.ime.unicamp.br/ matos

[46] M. C. Matos and L. Nachbin, Entire functions on locally convex spaces and convolution operators, Comp. Math. 44 (1981), 145-181.

[47] M. C. Matos and D. Pellegrino, Lorentz summing mappings, Math. Nachr. 283 (2010), 1409-1427.

[48] J. Mujica, Complex analysis in Banach spaces, North-Holland Mathematics Studies 120, North-Holland, 1986.

[49] S. Muro, D. Pinasco, M. Savransky, Strongly mixing convolution operators on Fréchet spaces of holomorphic functions, Integr. Equ. Oper. Theory 80 (2014), 453-468.

[50] L. Nachbin, Recent developments in infinite dimensional holomorphy, Bull. Amer. Math. Soc. 79 (1973), 625-639.

[51] L. Nachbin, Topology on Spaces of Holomorphic Mappings, Springer-Verlag, New York, 1969.

[52] H. Petersson, Hypercyclic subspaces for Fréchet space operators, J. Math. Anal. Appl. 319 (2006), 764-782.

[53] A. Pietsch, Operator Ideals, North-Holland, 1980.

[54] A. Pietsch, Ideals of multilinear functionals. In: Proceedings of the Second International Conference on Operator Algebras, Ideals and Their Applications in Theoretical Physics, pp.185-199, Teubner, 1983.

[55] H. H. Schaefer, Topological Vector Spaces, Springer-Verlag, 1971.

Vinícius V. Fávaro:

Faculdade de Matemática, Universidade Federal de Uberlândia, Uberlândia, MG, Cep: 38.400-902, Brazil, email: vvfavaro@gmail.com

Daniel Pellegrino:

Departamento de Matemática, Universidade Federal da Paraíba, João Pessoa, PB, Cep: 58.051-900, Brazil, email: dmpellegrino@gmail.com 